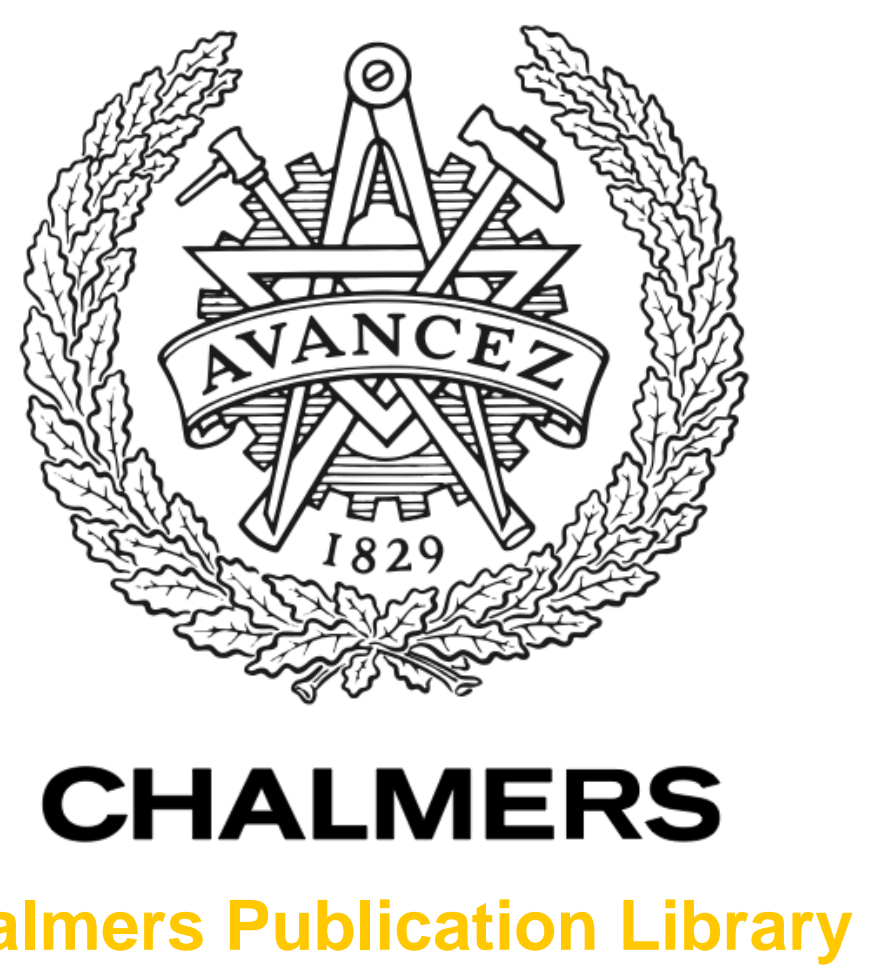

Challmers Publication Library

Extended Object Tracking using a Radar Resolution Model

This document has been downloaded from Chalmers Publication Library (CPL). It is the author's version of a work that was accepted for publication in:

IEEE Transactions on Aerospace and Electronic Systems (ISSN: 0018-9251)

Citation for the published paper:

Hammarstrand, L. ; Sandblom, F. ; Svensson, L. (2012) "Extended Object Tracking using a Radar Resolution Model". IEEE Transactions on Aerospace and Electronic Systems, vol. 48(3), pp. 2371 - 2386.

http://dx.doi.org/10.1109/TAES.2012.6237597

Downloaded from: http://publications.lib.chalmers.se/publication/161486

Notice: Changes introduced as a result of publishing processes such as copy-editing and formatting may not be reflected in this document. For a definitive version of this work, please refer to the published source. Please note that access to the published version might require a subscription.

Chalmers Publication Library (CPL) offers the possibility of retrieving research publications produced at Chalmers University of Technology. It covers all types of publications: articles, dissertations, licentiate theses, masters theses, conference papers, reports etc. Since 2006 it is the official tool for Chalmers official publication statistics. To ensure that Chalmers research results are disseminated as widely as possible, an Open Access Policy has been adopted.

The CPL service is administrated and maintained by Chalmers Library. 


\title{
Extended object tracking using a radar resolution model
}

\author{
Lars Hammarstrand, Fredrik Sandblom, Lennart Svensson Senior Member, IEEE, and Joakim Sörstedt
}

\begin{abstract}
This paper concerns the problem of vehicle tracking when multiple radar reflection centers could be resolved on each vehicle. For this extended target tracking problem we propose a radar sensor model, capable of describing such measurements, incorporating sensor resolution. Furthermore, we introduce approximations to handle the inherently complex data association problem. The evaluation in terms of describing measured data and resulting tracking performance shows that the model effectively exploits the information in multiple vehicle detections.
\end{abstract}

Index Terms-Radar, Sensor model, Extended targets, Tracking, Sensor resolution.

\section{INTRODUCTION}

A DVANCED automotive active safety systems often use sensors, such as radar and camera, to gather observations on the traffic environment around the vehicle. Through a tracking framework, these observations are refined to estimates of, e.g., position of other vehicles, pedestrians and the road. Based on the estimates, dangerous situations can be detected and decisions of appropriate actions are taken. For example, the system may warn the driver of an impending collision or intervene by braking or steering in order to avoid the collision or mitigate its consequences. For the active safety system to be able to make effective decisions, it is of great importance that the provided estimates meet the requirements in terms of accuracy and detail. To achieve this with a cost efficient system, the tracking framework needs to have an accurate description of the statistical properties of the sensor observations [2].

Many of the active safety systems on the market today are solely or partly radar based. Except from being robust against different weather conditions, the radar offers accurate measurements of range and range rate to objects. Furthermore, the radar has a long history of use in, e.g., airborne applications, and there exists a vast amount of literature on how to design a tracking system based on radar observations, see [3], [4] and the references therein. There are, however, important differences between target tracking in airborne applications

This work was sponsored by the Swedish Intelligent Vehicle Safety Systems (IVSS) program, and is a part of the SEnsor Fusion for Safety Systems (SEFS) project.

This article is a substantially expanded version of [1] presented at the IEEE symposium on Intelligent Vehicle Systems, Istanbul, Turkey, June 2007.

L. Hammarstrand and L. Svensson are with the Department of Signals and Systems, Chalmers University of Technology, Gothenburg, Sweden lars.hammarstrandechalmers.se., lennart.svensson@chalmers.se.

F. Sandblom is with Volvo 3P, Gothenburg, Sweden fredrik. sandblom. 2@volvo. com.

J. Sörstedt is with Volvo Car Corporation, Gothenburg, Sweden jsorstedevolvocars. com. and vehicle tracking for active safety systems. In airborne radar applications the aim is to track aircrafts at distances of tens of kilometers, whereas in automotive active safety systems the distances to the objects of interest are in the order of tens of meters. At such short distances, the radar resolution is typically finer than the physical extent of objects. Where, in airborne radar applications, the targets behave as point sources [3], [4], in automotive scenarios the radar is typically capable of detecting multiple features (reflection centers) on the same object. In the radar literature this type of target is referred to as an extended or distributed object/target [5].

Receiving multiple detections from a vehicle offers a possibility to extract detailed information about the object. For example, the spread of the individual detections gives information regarding the physical extent of the object as well as its orientation [2]. However, multiple measurements per object also introduce some considerable difficulties compared to the point source case. For one, the algorithms and models developed using the point source assumption are no longer valid. Additionally, an accurate sensor model is more complex as the detections are spread over large parts of the object and not accurately described as originating from a single point. The sensor model also needs to consider the possibility that a target can generate multiple detections in contrast to at most one in the point source case. The uncertainty in the number of target detections makes the data association problem more intricate. The aim of this paper is to develop a computationally tractable sensor model that accurately describes the radar detections from this type of object (vehicles). The ultimate purpose being to improve the tracking of vehicles for automotive active safety systems.

Although the classical point source assumption does not hold for extended objects, little attention has been given to find a more suitable tracking formulation. A good overview of different contributions up to 2004 can be found in [6], covering extended object tracking and the closely related problem of tracking groups of targets. More recent suggestions include, [7], [8] where a formal Bayesian tracking framework is proposed for estimating the centroid of the extended targets (or target groups). The object extension is modelled as an ellipse and it is assumed that multiple measurements can originate from each object. The elliptical shape of each object is described using a positive definite random matrix. By including these matrices in the state vector, both the target centroid and object extension are jointly estimated from data. Although the proposed approach shows promising results which are robust against object shape, it is difficult to exploit object specific shape information using this framework, when such information is available. Gilholm et. al. [9] propose a particle 
filter solution where the detections from the extended object are modelled by a non-homogenous Poisson point process with a known but arbitrary spatial intensity. Using this description, it is possible to include information about the shape of the objects, but due to the limited flexibility of the Poisson distribution, it is often impossible to incorporate specific knowledge regarding expected number of target returns. The probabilistic multi-hypothesis tracker (PMHT) [10] relaxes the point source criterion by modeling the measurement to target associations as stochastic and independent, and has been applied to extended object tracking in, e.g., [11]. Although the PMHT does not directly provide covariance estimates, the method is useful if the number of detections originating from each target cannot be accurately modeled.

For the problem of tracking vehicles using radar observations, there are reasons to believe that both the spatial distribution and the number of detections from a vehicle can be accurately modelled. For example, the study in [12] indicates that radar return from vehicles mainly originate from a number of specifically strong reflection centers (point sources), such as the headlamps and the wheel housings, see Fig. 3. Furthermore, if reflection centers are located within a resolution cell, the echoes from these reflectors are merged into a single joint detection (cluster detection). In [13]-[16], a model that captures the general behavior of a detection from a cluster including two sources/targets is used in conjunction with a description of the probability that the two targets are unresolved. Using this probabilistic description, the data association hypotheses and measurement model are expanded to also consider a merged detection from the two targets. A similar approach is proposed in [17], using a Gaussian approximation of the two-source cluster detection density originally derived in [18]. Although the solutions referred to here consider the influence of merged measurements, they are limited to handle only two sources, and the result is not easily expanded to the more general case of merging multiple sources.

Inspired by the findings in [12], we propose a radar sensor model describing the spatial distribution of vehicle detections as well as a probabilistic description over the number of vehicle detections. The proposed model also considers the effects of merging a general number of target reflections (limited resolution). As such, we are able to both incorporate shape information and expected number of vehicle detections, as well as describe the statistical behavior of the measurements. More specifically, the model family describes the radar reflections from a vehicle as originating from a set of reflection centers and, depending on the resolution of the sensor, reflectors likely to render a merged detection are grouped. The number of detections from each group is modelled as well as the distribution of the resulting detections. By associating measurements to reflector groups, instead of individual reflectors or reflector clusters, the number of association hypotheses is significantly reduced.

Furthermore, we derive a vehicle tracking framework based on our proposed sensor model. The framework is based on a linear minimum mean square error (LMMSE) estimator where the needed densities are estimated using the unscented transform (UT) [19]. A generalized version of the joint probabilistic data association (JPDA) technique [3], [20] is used to handle data association uncertainties. The proposed model is compared to the commonly used point source model in two aspects: model likelihood and tracking performance. The evaluation clearly indicates that the proposed model has significant benefits in both aspects.

The paper is organized as follows. In Section II the tracking problem is formalized and the necessary notation is introduced. Section III presents the radar sensor model, and in Section IV we show how this model can be used in a tracking framework. Finally, Section V presents evaluation results of our proposed sensor model and the derived tracking framework.

\section{Problem Formulation}

This article studies the problem of tracking vehicles with known physical dimensions, using multiple radars mounted on the host vehicle. The objective is twofold. First, to derive a family of detailed statistical models describing the radar returns from the vehicles. Second, to develop a vehicle tracking framework based on this model with the ultimate aim to improve the estimates of, i.e., the position and the velocity of the vehicles.

This section is partitioned as follows. The state parameters to be estimated are defined in Section II-A together with a model of how they evolve over time. Section II-B describes the necessary background properties of the radar observations, and Section II-C discusses in more detail the needed properties of the radar sensor model and the tracking framework for our specific problem.

\section{A. State parametrization}

All the parameters of interest are collected in the discrete time state vector $\mathbf{z}_{k}$, where $k$ is the discrete time index corresponding to continues time instance $t_{k}$. The state vector contains both the states of the surrounding vehicles and the host vehicle. Each vehicle, $l$, is described by the sub-state vector

$$
\mathbf{z}_{k}^{l}=\left[\zeta_{x, k}^{l} \zeta_{y, k}^{l} \psi_{k}^{l} v_{k}^{l} c_{k}^{l} \dot{v}_{k}^{l}\right]^{T},
$$

where $\left(\zeta_{x, k}^{l}, \zeta_{y, k}^{l}\right)$ is the position of vehicle $l$ expressed in a global Cartesian coordinate system. As illustrated in Fig. 1, $\Psi_{k}^{l}$ is the heading angle and $v_{k}^{l}$ is the speed in the heading direction of vehicle $l$ and $\dot{v}_{k}^{l}$ is its time derivative. The variable $c_{k}^{l}$ represents the curvature of the current path of the $l^{\text {th }}$ vehicle. The state vectors of all vehicles are stacked to form the complete state vector

$$
\mathbf{z}_{k}=\left[\begin{array}{lll}
\left(\mathbf{z}_{k}^{h}\right)^{T} & \left(\mathbf{z}_{k}^{1}\right)^{T}\left(\mathbf{z}_{k}^{2}\right)^{T} \ldots\left(\mathbf{z}_{k}^{N_{v}}\right)^{T}
\end{array}\right]^{T},
$$

where $\mathbf{z}_{k}^{h}$ is the host vehicle state and $N_{v}$ is the number of surrounding vehicles.

The state vector evolves over time as stipulated by the motion model,

$$
\mathbf{z}_{k}=\mathbf{f}_{k-1}\left(\mathbf{z}_{k-1}, \mathbf{e}_{k-1}\right),
$$




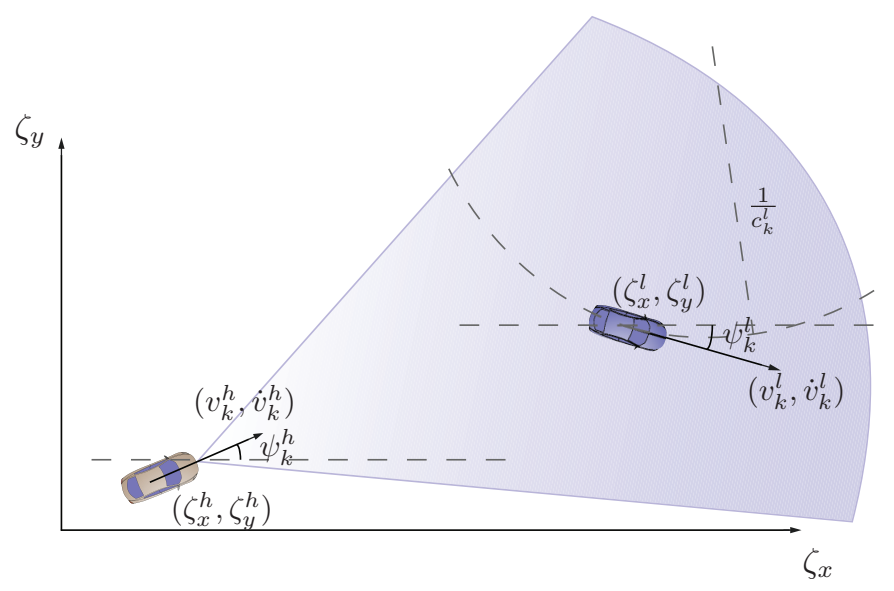

Figure 1. Coordinate system and state parametrization used in this paper.

where $\mathbf{f}_{k-1}(\cdot)$ is a non-linear function and $\mathbf{e}_{k}$ is a noise process included to reflect both model uncertainties and the dynamics of the vehicles. Assuming that the vehicles move independently, we can consider the motion of each vehicle separately. To describe the motion of a vehicle we use a slightly modified version of the simplified bicycle model derived in [1], where the difference lies in the use of curvature instead of yaw-rate, $\dot{\psi}_{k}=v_{k} c_{k}$.

\section{B. Radar observations}

For each discrete time $k$ a radar provides $M_{k}$ detections, where $M_{k}^{t}$ detections originate from the tracked vehicles and $M_{k}^{c}$ are clutter detections. All detections are stored in the unordered (unlabeled) measurement vector,

$$
\mathbf{y}_{k}=\left[\begin{array}{lll}
\left(\mathbf{y}_{k}^{1}\right)^{T} & \left(\mathbf{y}_{k}^{2}\right)^{T} \ldots\left(\mathbf{y}_{k}^{M_{k}}\right)^{T}
\end{array}\right]^{T} .
$$

Each detection is defined as

$$
\mathbf{y}_{k}^{i}=\left[r_{k}^{i} \dot{r}_{k}^{i} \phi_{k}^{i}\right]^{T},
$$

where $r_{k}^{i}$ is related to the range, $\dot{r}_{k}^{i}$ to the range rate, and $\phi_{k}^{i}$ to the angle to the object that gave rise to the detection relative to the sensor.

Let us define an ordered collection of the detections originating from the tracked vehicles as $\mathbf{y}_{k}^{t}$ and a collection of those originating from clutter as $\mathbf{y}_{k}^{c}$. These vectors are related to the measurement vector, $\mathbf{y}_{k}$, through an unknown permutation matrix, $\Pi_{p}^{M_{k}}$, with dimension $\left[M_{k} \mathbf{x} M_{k}\right]$,

$$
\mathbf{y}_{k}=\left(\boldsymbol{\Pi}_{p}^{M_{k}} \otimes \mathbf{I}_{3 \times 3}\right)\left[\begin{array}{l}
\mathbf{y}_{k}^{c} \\
\mathbf{y}_{k}^{t}
\end{array}\right] .
$$

where $\otimes$ is the Kronecker product and $\mathbf{I}_{3 \times 3}$ is a three-bythree identity matrix. The purpose of $\Pi_{p}^{M_{k}}$ is to describe mathematically that the measurement origin (data association) is unknown. In our model, all permutation matrices $\boldsymbol{\Pi}_{p}^{M_{k}}$ are equally likely, which means that the order of the detections in $\mathbf{y}_{k}$ is completely unknown (random). The treatment of this uncertainty is an important part in the derivation of the tracking framework and is further detailed in Section IV. However, let us first define $\mathbf{y}_{k}^{c}$ and $\mathbf{y}_{k}^{t}$ in more detail.
1) Clutter detections: It is commonly assumed, see e.g. [3], that $\mathbf{y}_{k}^{c}$ is described by a homogenous Poisson process in the observation space according to

$$
\mathbf{y}_{k, i}^{c} \sim \operatorname{Uniform}(V), \quad M_{k}^{c} \sim \operatorname{Poisson}(\mu V),
$$

where $\mathbf{y}_{k, i}^{c}$ is the $i^{t h}$ clutter measurement, $\mu$ is the clutter intensity and $V$ is the volume of the observation space. In addition, we assume that the clutter detections are independent from each other and the target detections.

2) Target detections: Given $\mathbf{z}_{k}$, we assume it is possible to partition the visible reflections centers into $N_{k}^{g}$ well separated groups, where each group can render multiple detections. Furthermore, we assume that the number of target detections for group $n, M_{k, n}^{t}$, has a probability mass function

$$
\operatorname{Pr}\left\{M_{k, n}^{t} \mid \mathbf{z}_{k}\right\} \text {. }
$$

that we can model. Conditioned on $M_{k, n}^{t}$, the detections from group $n$ can be described using a sensor model

$$
\mathbf{y}_{k, n}^{t}=\mathbf{h}_{k}^{n}\left(\mathbf{z}_{k}, \mathbf{w}_{k}, M_{k, n}^{t}\right),
$$

where $\mathbf{w}_{k}$ is a measurement noise process capturing both model uncertainties and measurement disturbances. From (9) we can generate

$$
\left.\mathbf{y}_{k}^{t}=\left[\begin{array}{lll}
\left(\mathbf{y}_{k, 1}^{t}\right)^{T} & \left(\mathbf{y}_{k, 2}^{t}\right)^{T} & \ldots \\
\mathbf{y}_{k, N_{k}^{g}}^{t}
\end{array}\right)^{T}\right]^{T},
$$

and the total number of target detections is given by

$$
M_{k}^{t}=\sum_{n=1}^{N_{k}^{g}} M_{k, n}^{t}
$$

\section{Objectives}

The main objective of this paper is to improve tracking performance by accurately modeling the radar response from the vehicles. To accomplish this, we need to derive an accurate model of the radar detections which is also suitable to be used in a tracking framework. In this section we discuss the objectives of the radar sensor model and the vehicle tracking framework separately. 
1) Sensor model: The aim of the sensor model is to describe the statistical behavior of the measurements, given $\mathbf{z}_{k}$. The behavior of the clutter detections is readily given by (7), but modelling the target detections (8) - (9) is more complicated. It is crucial that these models capture the behavior of the vehicle detections from different aspect angles and at all ranges [21]. A vehicle radar response model, i.e., expressions for (8) - (9), that considers these aspects is derived in Section III.

2) Tracking framework: Assuming that the number of vehicles is known, the objective of the tracking filter is to recursively calculate the posterior probability density function (pdf) $p\left(\mathbf{z}_{k} \mid \mathbf{Y}_{k}\right)$, where $\mathbf{Y}_{k} \triangleq\left\{\mathbf{y}_{1}, \mathbf{y}_{2}, \ldots, \mathbf{y}_{k}\right\}$ contains all the available observations up to and including time $k$. From $p\left(\mathbf{z}_{k} \mid \mathbf{Y}_{k}\right)$, it is then possible to compute estimates and uncertainty measures of $\mathbf{z}_{k}$. The calculation of $p\left(\mathbf{z}_{k} \mid \mathbf{Y}_{k}\right)$ is feasible if we have knowledge regarding two specific models [3], namely the motion model, defined in (3) and the sensor model, defined by (7) - (9).

To arrive at a computationally tractable solution, we restrict our tracking filter to an LMMSE estimator of $\mathbf{z}_{k}$. As such, only the first two moments of $p\left(\mathbf{z}_{k} \mid \mathbf{Y}_{k}\right)$ need to be calculated, i.e.,

$$
\hat{\mathbf{z}}_{k \mid k}=\mathrm{E}\left\{\mathbf{z}_{k} \mid \mathbf{Y}_{k}\right\}, \quad \hat{\mathbf{P}}_{k \mid k}=\operatorname{Cov}\left\{\mathbf{z}_{k} \mid \mathbf{Y}_{k}\right\} .
$$

However, due to non-linearities in both the process and measurement model it is difficult to find an exact solution to (12). Instead, filters which approximate these moments are commonly used, e.g., the extended Kalman filter (EKF) [22] or the unscented Kalman filter (UKF) [19]. The latter is derived for the proposed sensor model in Section IV, treating the uncertainty in measurement origin (data association) modelled by the unknown permutation matrix $\Pi_{p}^{M_{k}}$.

\section{RADAR SENSOR MODEL}

Our proposed sensor model is based on the findings presented in [12], where the radar response from vehicles is modeled as originating from reflection centers (features) on the vehicles more likely to reflect the incident radar wave. Due to limitations in radar signal bandwidth, pulse duration and antenna aperture size, radar sensors are not capable of resolving reflection centers that are too closely spaced. As such, not all of these reflectors are always resolvable and the response from some might merge to form a joint detection. In [12], a mapping is proposed for how to transform the vehicle states to a set of reflector positions in observation space. Additionally, a scheme is described for how to form clusters of those reflection centers that are unresolvable and how to model detections from these clusters. As this model was developed for simulation purposes, rather than for use in a tracking system, it neglects important probabilistic descriptions needed in the tracking context. For example, the model requires the received signal strength of each individual reflection center to be known. Moreover, conditioned on the signal strength and $\mathbf{z}_{k}$, both which reflectors are clustered and the positions of the resulting clusters are deterministic. In the tracking context, it is not realistic that the signal strength is known a-priori and consequently, we do not know which reflectors are clustered or the position of the resulting detections from the vehicle.
In this section, we derive a radar sensor model using a stochastic description of the received signal amplitude from each reflector center, arriving at a model more suitable in a tracking framework. The derivation is conducted in four steps which are shown in Fig. 2 and summarized as follows. First, based on the model in [12], the positions of the reflection centers of the vehicles in $\mathbf{z}_{k}$ are mapped to the observation space. Second, we form all possible clusters of reflection centers which may generate merged detections. Due to uncertainty regarding which reflectors are resolved and which are not, the resulting probability density of reflector cluster could be highly multi-modal. Third, to alleviate this multi-modality, we group reflectors which may belong to the same cluster, and approximate the cluster density by marginalizing over all cluster possibilities. The result is a description of reflector groups capable of generating multiple measurements. Finally, depending on the probability of detecting the possible clusters in each group, we find an expression for (8). Assuming that the measurement noise is additive and Gaussian, we now write (9) on the form

$$
\mathbf{y}_{k, n}^{t}=\mathcal{G}_{n}\left(\mathbf{z}_{k}, M_{k, n}^{t}\right)+\mathbf{w}_{k}, \mathbf{w}_{k} \sim \mathcal{N}\left(\mathbf{0}, \mathbf{W}_{k}^{M_{k, n}^{t}}\right)
$$

where

$$
\mathbf{W}_{k}^{M_{k, n}^{t}}=\mathbf{I}_{M_{k, n}^{t} \mathrm{x} M_{k, n}^{t}} \otimes \mathbf{W}_{k} .
$$

The function $\mathcal{G}_{n}(\cdot)$ maps $\mathbf{z}_{k}$ to the target measurement vector for group $n$, given knowledge regarding the number of measurements generated by the group, $M_{k, n}^{t}$. Note that although we condition on $\mathbf{z}_{k}$ and the number of detections from the group, $\mathcal{G}_{n}\left(\mathbf{z}_{k}, M_{k, n}^{t}\right)$ is still stochastic due to uncertainty in which reflectors that are clustered (we call this clustering uncertainty).

The following sections present the derivation of the distribution of $\mathcal{G}_{n}\left(\mathbf{z}_{k}, M_{k, n}^{t}\right)$ and $M_{k, n}^{t}$ using the steps described above. To simplify notation, the time dependence is omitted and all stochastic variables are conditioned on $\mathbf{z}_{k}$, even though it is not explicitly stated.

\section{A. Reflection center model}

According to the model in [12], the studied radar only receives reflections from a discrete set of points on a vehicle, so called reflection centers. The different reflection centers are divided into two categories: point reflectors and plane reflectors. Fig. 3 displays the configuration of point reflectors suggested in [12], where the reflectors are placed in the vehicle wheel houses and corners. Associated with each reflector is a visibility region, indicated by cones in Fig. 3; a reflector can only render a reflection if the sensor is within this region. The plane reflectors are modelled as circle sectors typically describing the sides of the vehicle. Furthermore, it is assumed that the radar only can receive a reflection from these plane reflector if there is a point on the surface which normal points directly towards the sensor. The reflecting point on a surface therefore depends on the position of the sensor, and may change over time as the vehicle moves relative to the sensor platform. 


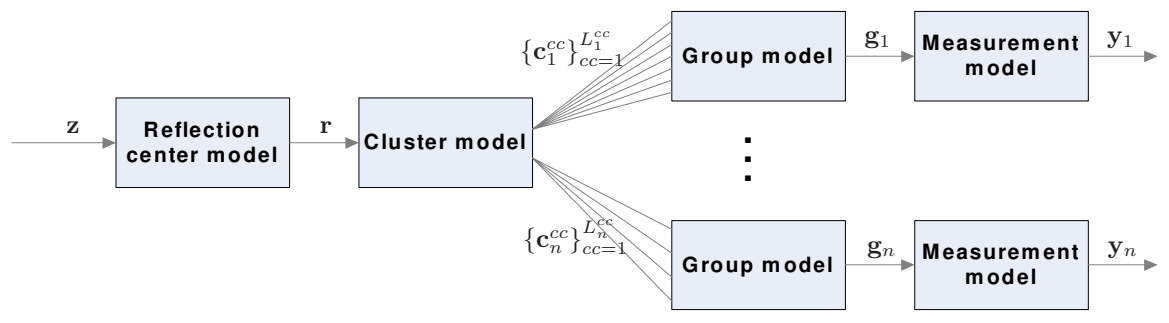

Figure 2. Schematic view of the measurement generation process in our proposed radar sensor model. The notation used in the figure is introduced in subsequent sections.

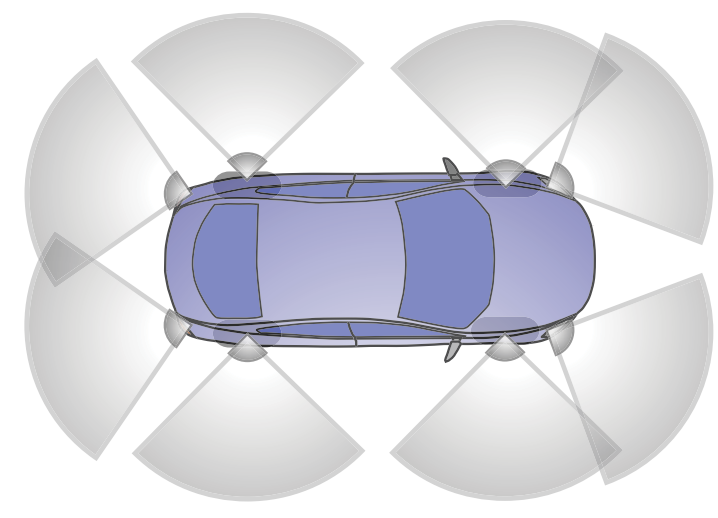

Figure 3. Vehicle reflection centers with associated visibility regions.

Given the vehicles' positions and physical dimensions, each reflector $i$ has a deterministic position in observation space, denoted $\mathbf{r}_{i}=\left[r_{i}, \dot{r}_{i}, \phi_{i}\right]^{T}$ and expected signal power $\sigma_{i}$, expressed as

$$
\left[\left(\mathbf{r}_{i}\right)^{T} \sigma_{i}\right]^{T}=\mathcal{R}_{i}(\mathbf{z})
$$

where the mapping $\mathcal{R}$ is defined in Appendix A. Although the physical dimensions of the observed objects are assumed known in this paper, in a sensor data fusion system, information regarding object extent could be provided by, e.g., a vision sensor and/or vehicle-to-vehicle communication.

In addition to the position of the reflector in observation space, it is also important to model the signal amplitude, $A_{i}$, of the received reflection. This is an important model feature as the probability of detecting a reflector depends on the strength of the received echo, and the position of a merged detection depends on the relative amplitude of the included echoes. The amplitude model used in [12] is a deterministic function of the radar antenna pattern as well as the position and visibility of the reflectors. We instead propose to use a Swerling I model [23] for the amplitude of the reflected signal, where the reflection amplitudes are modeled as fluctuating according to the Rayleigh distribution,

$$
A_{i} \sim \operatorname{Rayleigh}\left(\sigma_{i}\right)
$$

As is shown in the coming section, using a stochastic amplitude model instead of a deterministic enables us to describe uncertainty regarding number of vehicle detections as well as their positions.

\section{B. Cluster model}

In Section III-A we presented a model for the vehicle response from a radar with infinite resolution through the mapping $\mathbf{z} \stackrel{\mathcal{R}}{\longrightarrow} \mathbf{r}$. However, a sensor with limited resolution cannot resolve too closely spaced reflectors. To model this behavior, a resolution cell is used

$$
\Delta_{\mathbf{d}}=\left[\Delta_{r} \Delta_{\dot{r}} \Delta_{\phi}\right]^{T}
$$

and two radar responses which are not separated more than $\boldsymbol{\Delta}_{\mathrm{d}}$, in all three dimensions, yield a merged detection. Unfortunately, the situation is more complicated for multiple reflectors, as unresolved clusters can be formed in several ways.

1) Cluster formation: In [12], the following algorithm is used to map reflectors into clusters, an operation here denotes as $\mathbf{r} \stackrel{\mathcal{C}}{\rightarrow} \mathbf{c}^{c c}$ :

i) Find the reflector with the strongest amplitude, $A_{i}$.

ii) Form a cluster by identifying the reflectors which are within the resolution cell (centered at $\mathbf{r}_{i}$ ).

iii) Repeat $i$ ) and $i i$ ) with the remaining reflectors, until no reflectors are left.

The clustering algorithm above, can be used to divide the set of all visible reflectors into clusters and we refer to a description of all resulting clusters as a cluster constellation. However, it is important to note that since the amplitudes of the reflections are stochastic (in contrast to [12]), several different cluster constellations may be possible, even for a given z. For notation, we construct a list of all possible constellations, and introduce the variable $c c$ as a pointer to the cluster constellations in that list. The total number of constellations in the list is denoted $N^{c c}$ (and consequently $c c \in\left\{1,2, \ldots, N^{c c}\right\}$ ), and the number of reflector clusters in constellation $c c$ is $L^{c c}$. Fig. 4 illustrates these concepts by showing three possible cluster constellations in a simple example. Here, $N^{c c}=3$ with $L^{1}=1, L^{2}=2$ and $L^{3}=2$ as the first constellation clusters all reflectors and the other two contains two clusters each.

Cluster $i$ in constellation $c c$, containing $J$ reflectors ${ }^{1}$ with indices $i_{1}, \ldots, i_{J}$, can at most generate one detection which in that case can be modelled as $\mathbf{y}_{i}^{c c}=\mathbf{c}_{i}^{c c}+\mathbf{w}_{i}^{c c}$ where $\mathbf{w}_{i}^{c c} \sim \mathcal{N}(\mathbf{0}, \mathbf{W})$. The signal component, $\mathbf{c}_{i}^{c c}$, is modelled

\footnotetext{
${ }^{1}$ The notation for the number of reflectors will change as we can get more specific. In this section we use $J$ to indicate the number of reflectors in a generic cluster.
} 

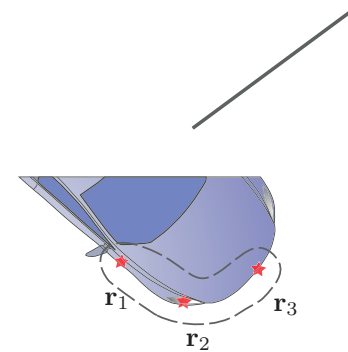

$c c=1$
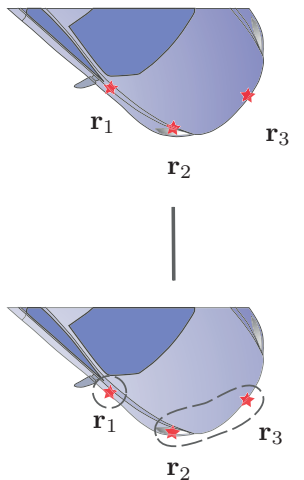

$c c=2$
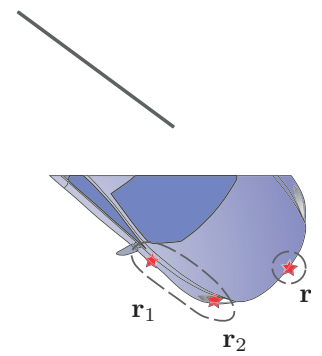

$c c=3$

Figure 4. Example of a vehicle with three reflectors (top) and three different cluster constellations (bottom). The dashed line corresponds to a reflector cluster.

as a weighted sum [12] of reflector components:

$$
\mathbf{c}_{i}^{c c}=\sum_{l=1}^{J} w_{i_{l}} \mathbf{r}_{i_{l}}
$$

where

$$
w_{i_{l}}=\frac{A_{i_{l}}}{\sum_{l=1}^{J} A_{i_{l}}} .
$$

Although this is a rather simplified model of the underlying physical phenomenon of merged measurements (such as target glint) [5], [24], it serves the purposes for our radar sensor model. Since the amplitudes are stochastic, so are the weights (19) and the signal component of the cluster (18). As for the reflector detections, the received amplitude of a cluster is also Rayleigh distributed but with the parameter

$$
\sigma_{i}^{c c}=\sqrt{\sum_{l=1}^{J} \sigma_{i_{l}}^{2}} .
$$

2) Cluster density: For a cluster, the distribution of its position is defined by (16), (18) - (19), which is difficult to evaluate. As the aim is to use the proposed sensor model in a Kalman filter framework, it is convenient to approximate $p\left(\mathbf{c}_{i}^{c c} \mid \mathbf{z}\right)$ as a Gaussian density with the same first two moments as the underlying distribution.

Let overscore denote the expected value of stochastic variables, such that, e.g., $\bar{A}_{i}=E\left\{A_{i}\right\}$. Further, let $\Delta \mathbf{r}_{i_{l}}=$ $\mathbf{r}_{i_{l}}-\overline{\mathbf{c}}_{i}^{c c}, \Delta w_{i_{l}}=w_{i_{l}}-\bar{w}_{i_{l}}$ and set $S_{i}=\sum_{l=1}^{J} A_{i_{l}}$. The first moment of $\mathbf{c}_{i}^{c c}$, as given by (18), is

$$
\overline{\mathbf{c}}_{i}^{c c}=\sum_{l=1}^{J} \bar{w}_{i_{l}} \mathbf{r}_{i_{l}}
$$

and after some manipulations an expression for the covariance can be found as

$$
\mathbf{C}_{i}^{c c}=\sum_{s, t=1}^{J} \Delta \mathbf{r}_{i_{s}}\left(\Delta \mathbf{r}_{i_{t}}\right)^{T} E\left\{\Delta w_{i_{s}} \Delta w_{i_{t}}\right\} .
$$

The position of each reflector, $\mathbf{r}_{i_{l}}$, is given by transformation (15), but we also need to express $\bar{w}_{i_{l}}$ and $\operatorname{Cov}\left\{w_{i_{s}}, w_{i_{t}}\right\}$. As the moments of a Rayleigh distribution are well known, approximations of these quantities are readily found through Taylor expansion,

$$
w_{i_{l}}=\frac{A_{i_{l}}}{S_{i}} \approx \frac{\bar{A}_{i_{l}}}{\bar{S}_{i}}+\frac{A_{i_{l}}}{\bar{S}_{i}}-\frac{S_{i} \bar{A}_{i_{l}}}{\bar{S}_{i}^{2}} .
$$

More details on the derivation of the mean and covariance of $\mathbf{c}_{i}^{c c}$ as well as the approximations used are found in Appendix B.

To summarize, we propose a stochastic mapping of reflectors into cluster constellations, $\mathbf{r} \stackrel{\mathcal{C}(\cdot)}{\longrightarrow}\left\{\mathbf{c}^{c c}\right\}_{c c=1}^{N^{c c}}$, where $N^{c c}$ is deterministic but both $c c$ and $\mathbf{c}^{c c}=\left[\mathbf{c}_{1}^{c c}, \ldots, \mathbf{c}_{L^{c c}}^{c c}\right]$ are stochastic. The density of each cluster in each constellation, $\mathbf{c}_{i}^{c c}$, is approximated as a Gaussian density,

$$
p\left(\mathbf{c}_{i}^{c c} \mid c c, \mathbf{z}\right)=\mathcal{N}\left(\mathbf{c}_{i}^{c c} ; \overline{\mathbf{c}}_{i}^{c c}, \mathbf{C}_{i}^{c c}\right),
$$

where $\overline{\mathbf{c}}_{i}^{c c}$ and $\mathbf{C}_{i}^{c c}$ are given by (21) and (22), respectively.

\section{Group model}

In multi-target scenarios, the total number of possible clusters, $\sum_{c c=1}^{N^{c c}} L^{c c}$, can be significant. Hence, it could be difficult to find a computationally feasible solution for associating measurements to individual clusters. To mitigate this difficulty, we suggest to form reflector groups containing reflectors that are likely to get clustered, and describe the measurement distribution by marginalizing over the cluster constellations. Let a group be a set of reflectors, formed such that for every reflector $i$ in the group, all other reflectors belonging to one or more clusters with reflector $i$ are also included. As a consequence, each reflector $i$ in the group is positioned within $\Delta_{\mathrm{d}}$ to at least one other reflector in the group. The number of groups, $N^{g}$, is then the total number of such partitions of the reflectors. Fig. 5 displays a scenario where two groups, i.e., $N^{g}=2$, are formed; one containing only one reflection center in the rear wheel and the other composed of three reflection centers in the front. A suboptimal, but simplified, solution to the association problem is obtained by associating the detections to the reflector groups. By ignoring which specific cluster in a group that gave rise to a detection, the number of hypotheses are reduced substantially.

Each group is viewed as an entity which can generate multiple and independent detections. The number of detections 


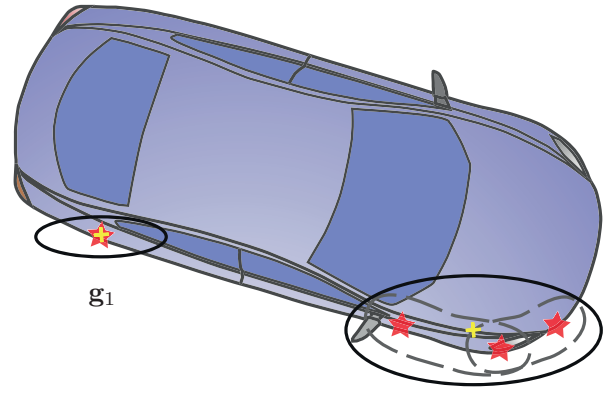

$\mathbf{g}_{2}$

Figure 5. The formation of two reflector groups (solid lines).

from group $n$ is denoted by $M_{n}^{t}$, and the signal components (the positions) as $\tilde{\mathbf{g}}_{n}=\left[\left(\mathbf{g}_{n, 1}\right)^{T}, \ldots,\left(\mathbf{g}_{n, M_{n}^{t}}\right)^{T}\right]^{T}$. Using this notation, (13) can be written as

$$
\mathbf{y}_{n}^{t}=\tilde{\mathbf{g}}_{n}+\mathbf{w}
$$

As only reflectors within each group can form clusters with each other, we can consider the cluster constellations for each group independently. For group $n$ we can generate $N_{n}^{c c}$ cluster constellations, and we let $c c$ in this case indicate one specific constellation in this group and $L_{n}^{c c}$ denote the number of clusters in this cluster constellation. A new list of cluster constellations is generated for each group and $c c$ is used to index one of the constellations. Further, let $\mathbf{c}_{n, l}^{c c}$ denote the signal component of the $l^{\text {th }}$ cluster in constellation $c c$ for group $n$, and $P_{n}^{c c}(l)$ denote the detection probability of this cluster - a probability easily computed from the Rayleigh assumption in (16) and (20). If we assume that all possible cluster constellations are equally likely, we can describe the pdf of $\tilde{\mathbf{g}}_{n}$ by approximating its components $\mathbf{g}_{n, i}$ as independent and identically distributed with the density ${ }^{2}$

$$
p\left(\mathbf{g}_{n, i}\right)=\frac{1}{N_{n}^{c c}} \sum_{c c=1}^{N_{n}^{c c}} \sum_{l=1}^{L_{n}^{c c}} q_{n, l}^{c c} p_{\mathbf{c}_{n, l}^{c c}}\left(\mathbf{g}_{n, i}\right),
$$

where the weights $q_{n, l}^{c c}$ are defined as

$$
q_{n, l}^{c c}=\frac{P_{n}^{c c}(l)}{\sum_{m=1}^{L_{n}^{c c}} P_{n}^{c c}(m)} .
$$

To further simplify the implementation of a tracking algorithm based on this model we make a Gaussian approximation, $p\left(\mathbf{g}_{n, i}\right) \approx \mathcal{N}\left(\mathbf{g}_{n, i} ; \overline{\mathbf{g}}_{n, i}, \mathbf{C}_{n}\right)$. Using the approximation in (24), the expected value, $\overline{\mathbf{g}}_{n}=\mathrm{E}\left\{\mathbf{g}_{n, i}\right\}$ is given by

$$
\overline{\mathbf{g}}_{n}=\frac{1}{N_{n}^{c c}} \sum_{c c=1}^{N_{n}^{c c}} \sum_{l=1}^{L_{n}^{c c}} q_{n, l}^{c c} \overline{\mathbf{c}}_{n, l}^{c c}
$$

and the second moment, $\mathbf{C}_{n}=\mathrm{E}\left\{\left(\mathbf{g}_{n, i}-\overline{\mathbf{g}}_{n}\right)\left(\mathbf{g}_{n, i}-\overline{\mathbf{g}}_{n}\right)^{T}\right\}$ by

$$
\mathbf{C}_{n}=\sum_{c c=1}^{N_{n}^{c c}} \sum_{l=1}^{L_{n}^{c c}} \frac{q_{n, l}^{c c}}{N_{n}^{c c}}\left(\mathbf{C}_{n, l}^{c c}+\left(\overline{\mathbf{g}}_{n}-\overline{\mathbf{c}}_{n, l}^{c c}\right)\left(\overline{\mathbf{g}}_{n}-\overline{\mathbf{c}}_{n, l}^{c c}\right)^{T}\right),
$$

\footnotetext{
${ }^{2}$ In (26), the notation $p_{\mathbf{c}_{n, l}^{c c}}\left(\mathbf{g}_{n, i}\right)$ should be interpreted as the pdf of $\mathbf{c}_{n, l}^{c c}$ evaluated at $\mathbf{g}_{n, i}$.
}

where $\overline{\mathbf{c}}_{n, l}^{c c}$ and $\mathbf{C}_{n, l}^{c c}$ is given by (21) and (22), respectively.

Additionally, to complete the description of the groups, we need to calculate the probability mass function $M_{n}^{t}$, the number of detections originating from group $n$. By again assuming that all cluster constellations are equally likely, we have

$$
\operatorname{Pr}\left\{M_{n}^{t}\right\}=\frac{1}{N_{n}^{c c}} \sum_{c c=1}^{N_{n}^{c c}} \operatorname{Pr}\left\{M_{n}^{t} \mid c c\right\},
$$

where $\operatorname{Pr}\left\{M_{n}^{t} \mid c c\right\}$ is easily calculated from $P_{n}^{c c}(l)$.

In summary; we group closely spaced reflectors and use the cluster description to calculate the expected signal component each group, its covariance matrix and the probability mass function for $M_{n}^{t}$, the number of detections from group $n$.

\section{Target measurement model}

In Sections III-A, III-B and III-C we describe three mappings, $\mathcal{R}(\cdot), \mathcal{C}(\cdot)$ and $\mathcal{G}(\cdot)$, relating the position of the vehicles to the measurement distribution. The procedure can be depicted as

$$
\mathbf{z} \stackrel{\mathcal{R}(\cdot)}{\longrightarrow} \mathbf{r} \stackrel{\mathcal{C}(\cdot)}{\longrightarrow}\left\{\mathbf{c}^{c c}\right\}_{c c=1}^{N^{c c}} \stackrel{\mathcal{G}(\cdot)}{\longrightarrow} \mathbf{g}_{n}, M_{n}^{t},
$$

where the first two mappings are deterministic whereas the last two are stochastic due to uncertainty in the resolution capabilities of the sensor. The transformation $\mathbf{z} \stackrel{\mathcal{R}(\cdot)}{\longrightarrow} \mathbf{r}$ describes the signal components of strong (vehicle related) radar reflectors in observation space. By modelling the resolution capability of the sensor, $\mathcal{C}(\cdot)$, we form a set of cluster constellations, $\left\{\mathbf{c}^{c c}\right\}_{c c=1}^{N^{c c}}$. To reduce the complexity of the data association problem, we form groups of reflectors that belong to the same cluster constellations, $\mathcal{G}(\cdot)$. The groups are described by their spatial density, $p\left(\mathbf{g}_{n}\right) \approx \mathcal{N}\left(\mathbf{g}_{n} ; \overline{\mathbf{g}}_{n}, \mathbf{C}_{n}\right)$, and the probability mass function $\operatorname{Pr}\left\{M_{n}^{t}\right\}$, describing the number of target detections from each group. The result is a description of target measurements originating from groups of reflectors, where each group $n$ generates $M_{n}^{t}$ independent and identically distributed measurements. The $i^{t h}$ detection from group $n$,

$$
\mathbf{y}_{n, i}^{t} \sim \mathcal{N}\left(\overline{\mathbf{g}}_{n}, \mathbf{C}_{n}+\mathbf{W}\right),
$$

is an independent of all other detections (conditioned on $\mathbf{z}$ ). The complete target measurement vector $\mathbf{y}^{t}$ can be generated by drawing the number of target detections from the group, $M_{n}^{t}$, according to (30), for each group $n=1 \ldots N^{g}$. Subsequently, construct $\mathbf{y}_{n}^{t}$ by generating $M_{n}^{t}$ independent realizations of $\mathbf{y}_{n, i}^{t}$ conforming to (31). The complete target measurement vector is formed by concatenating all group measurements as defined in (10). This concludes the derivation of our sensor model.

\section{TRACKING FRAMEWORK}

In this section we present a tracking framework for recursively calculating the posterior density, $p\left(\mathbf{z}_{k} \mid \mathbf{Y}_{k}\right)$, using the proposed sensor model derived in Section III. To handle uncertainty in the number of target and clutter detections as well as the random permutation matrix, $\Pi_{p}^{M_{k}}$, in the calculation of $p\left(\mathbf{z}_{k} \mid \mathbf{Y}_{k}\right)$, it is convenient to introduce a data 
association hypothesis vector, $\boldsymbol{\lambda}$. The purpose of this vector is to associate detection number $j$ in $\mathbf{y}_{k}$ to a certain group, $n$. Consequently, $\boldsymbol{\lambda}(j)=n$, if measurement $j$ originates from group $n$ and, $\boldsymbol{\lambda}(j)=0$, if it is to be regarded as clutter. Using this description, the sensor model can be written as

$p\left(\mathbf{y}_{k}^{j} \mid \boldsymbol{\lambda}, \mathbf{z}_{k}\right)= \begin{cases}\mathcal{N}\left(\mathbf{y}_{k}^{j} ; \overline{\mathbf{g}}_{\boldsymbol{\lambda}(j)}, \mathbf{C}_{\boldsymbol{\lambda}(j)}+\mathbf{W}_{k}\right) & \text { if } \boldsymbol{\lambda}(j) \neq 0 \\ \left(\frac{1}{V}\right) & \text { if } \boldsymbol{\lambda}(j)=0 .\end{cases}$

This formulation makes it possible to associate measurements to group $n$ according to the support of $\operatorname{Pr}\left\{M_{n}^{t} \mid \mathbf{z}\right\}$. That is, each group can generate multiple detections, each carrying information regarding the state of the vehicle. This distinguishes our sensor model from the classical point source model.

By considering all possible data association hypothesis, the posterior density can be formed as

$$
p\left(\mathbf{z}_{k} \mid \mathbf{Y}_{k}\right)=\sum_{\boldsymbol{\lambda}} p\left(\mathbf{z}_{k} \mid \boldsymbol{\lambda}, \mathbf{Y}_{k}\right) \operatorname{Pr}\left\{\boldsymbol{\lambda} \mid \mathbf{Y}_{k}\right\}
$$

where $p\left(\mathbf{z}_{k} \mid \boldsymbol{\lambda}, \mathbf{Y}_{k}\right)$ is the posterior density without data association uncertainty and $\operatorname{Pr}\left\{\boldsymbol{\lambda} \mid \mathbf{Y}_{k}\right\}$ is the probability of that association. Due to, e.g., non-linearities in (15) and the dimensionality of the data association problem, it is difficult to find an exact solution to (33). Instead, we resort to an approximate solution.

In the literature it is possible to find several possible approaches, such as particle filters [25], Multiple Hypothesis Tracking (MHT) filters [26] or the Probabilistic Multiple Hypothesis Tracker (РМHT) [10] to handle or simplify these types of problems. To make the implementation suitable for real-time applications with limited capacity to batch measurements, we propose a Kalman-like filter framework [27] employing a generalized version of the Joint Probabilistic Data Association (JPDA) algorithm [20]. The generalization of the JPDA algorithm for this problem consists in allowing multiple measurements to originate from the same group, in contrast to at most one in the original JPDA formulation. The conditional posterior density, $p\left(\mathbf{z}_{k} \mid \boldsymbol{\lambda}, \mathbf{Y}_{k}\right)$, can be calculated for each $\boldsymbol{\lambda}$, and we approximate this distribution by a Gaussian density with mean $\hat{\mathbf{z}}_{k \mid k}^{\lambda}$ and covariance $\mathbf{P}_{k \mid k}^{\boldsymbol{\lambda}}$. Estimates of $\hat{\mathbf{z}}_{k \mid k}^{\boldsymbol{\lambda}}$ and $\mathbf{P}_{k \mid k}^{\boldsymbol{\lambda}}$ are found using the UT [19] through

$$
\begin{aligned}
& \hat{\mathbf{z}}_{k \mid k}^{\boldsymbol{\lambda}}=\hat{\mathbf{z}}_{k \mid k-1}+\mathbf{P}_{\mathbf{z y}}^{\boldsymbol{\lambda}}\left(\mathbf{P}_{\mathbf{y y}}^{\boldsymbol{\lambda}}\right)^{-1}\left(\mathbf{y}_{k}^{\boldsymbol{\lambda}}-\hat{\mathbf{y}}_{k \mid k-1}^{\boldsymbol{\lambda}}\right) \\
& \mathbf{P}_{k \mid k}^{\boldsymbol{\lambda}}=\mathbf{P}_{k \mid k-1}-\mathbf{P}_{\mathbf{z y}}^{\boldsymbol{\lambda}}\left(\mathbf{P}_{\mathbf{y y}}^{\boldsymbol{\lambda}}\right)^{-1}\left(\mathbf{P}_{\mathbf{z y}}^{\boldsymbol{\lambda}}\right)^{T},
\end{aligned}
$$

where $\hat{\mathbf{z}}_{k \mid k-1}$ and $\mathbf{P}_{k \mid k-1}$ are estimates of the mean and covariance of $\mathbf{z}_{k} \mid \mathbf{Y}_{k-1}$, respectively. The covariance, $\mathbf{P}_{\mathbf{y y}}^{\boldsymbol{\lambda}}$, is the innovation covariance under the data association hypothesis $\boldsymbol{\lambda}$ and, $\mathbf{P}_{\mathbf{z y}}^{\boldsymbol{\lambda}}$ is the corresponding cross covariance between the state and the measurements. In accordance with the JPDA idea, the resulting Gaussian mixture (33) is also approximated as a single Gaussian where the contribution from the individual densities are weighted by their hypothesis probability, $\operatorname{Pr}\left\{\boldsymbol{\lambda} \mid \mathbf{Y}_{k}\right\}$. Given a Gaussian prior density, $p\left(\mathbf{z}_{k-1} \mid \mathbf{Y}_{k-1}\right)$, the proposed approach is briefly outlined below.

1) State prediction: Estimate $\hat{\mathbf{z}}_{k \mid k-1}$ and $\mathbf{P}_{k \mid k-1}$ by propagating $\mathbf{z}_{k-1} \mid \mathbf{Y}_{k-1}$ through the motion model (3) using the unscented transform.
2) Measurement prediction: Transform $\mathbf{z}_{k} \mid \mathbf{Y}_{k-1}$ to the observation space, using the series of mappings derived in Section III and the unscented transform to retain an approximation of the predicted group measurement

$$
\hat{\mathbf{y}}_{k, n}^{t}=\hat{\mathbf{g}}_{n}=\mathrm{E}\left\{\mathbf{g}_{n} \mid \mathbf{Y}_{k-1}\right\}
$$

as well as the predicted group covariances

$$
\begin{aligned}
\mathbf{P}_{\mathbf{g g}}^{n} & =\operatorname{Cov}\left\{\mathbf{g}_{n, i}, \mathbf{g}_{n, i} \mid \mathbf{Y}_{k-1}\right\}, \\
\mathbf{P}_{\mathbf{g g}}^{n m} & =\operatorname{Cov}\left\{\mathbf{g}_{n, i}, \mathbf{g}_{m, j} \mid \mathbf{Y}_{k-1}\right\},
\end{aligned}
$$

where $i \neq j$ if $n=m$. The innovation covariance, $\mathbf{P}_{\mathbf{y y}}^{\boldsymbol{\lambda}}$, is given by (37) - (38) and the measurement noise covariance, $\mathbf{W}_{k}$.

3) Data association: Use $\hat{\mathbf{g}}_{n}$ and $\mathbf{P}_{\mathbf{y y}}^{n}=\mathbf{P}_{\mathrm{gg}}^{n}+\mathbf{W}_{k}$ to perform measurement gating. Then generate the set of all possible measurement to group association hypotheses and calculate their probabilities $\operatorname{Pr}\left\{\boldsymbol{\lambda} \mid \mathbf{Y}_{k}\right\}$.

4) Measurement update: For all $\boldsymbol{\lambda}$, form the needed entities in (34) - (35) and approximate $p\left(\mathbf{z}_{k} \mid \boldsymbol{\lambda}, \mathbf{Y}_{k}\right)$. Finally, $p\left(\mathbf{z}_{k} \mid \mathbf{Y}_{k}\right)$, is found by marginalizing the data association hypotheses, see (33).

A detailed description of the different steps is given in the following sections.

\section{A. State prediction}

The state prediction is performed by calculating a Gaussian approximation of the predicted density

$$
p\left(\mathbf{z}_{k} \mid \mathbf{Y}_{k-1}\right)=\int p\left(\mathbf{z}_{k} \mid \mathbf{z}_{k-1}\right) p\left(\mathbf{z}_{k-1} \mid \mathbf{Y}_{k-1}\right) d \mathbf{z}_{k-1} .
$$

This approximation is found by propagating $p\left(\mathbf{z}_{k-1} \mid \mathbf{Y}_{k-1}\right)$ through (3) using the unscented transform [19] as

$$
p\left(\mathbf{z}_{k} \mid \mathbf{Y}_{k-1}\right) \approx \mathcal{N}\left(\mathbf{z}_{k} ; \hat{\mathbf{z}}_{k \mid k-1}, \mathbf{P}_{k \mid k-1}\right) .
$$

Assuming the vehicles move independently, the unscented transform can be performed for each vehicle separately.

\section{B. Measurement prediction}

In Section III we derived a radar sensor model conditioned on the state through a series of mappings. To form the expressions (34) - (35) we need estimates of the first and the second-order moments (36), (37) and (38). Again we use the unscented transform to find approximations of these moments. However, for our proposed group measurement model, the approximation is not as straightforward as for the motion model. As such, it requires some additional discussion.

Using the unscented transform described in [19], we choose $2 n_{z}+1$ deterministic sigma points with associated weights, where $n_{z}$ is the dimensionality of $\mathbf{z}_{k}$. The sigma points and their weights are chosen such that they capture the first two moments of (40) exactly. Let us denote the set of sigma points with associated weights as,

$$
\left\{\mathbf{Z}_{k}^{i}, u_{k}^{i}\right\}_{i=1}^{2 n_{z}+1}
$$


Although not required, we choose the weights such that $u_{k}^{i}>0, \forall i=1 \ldots 2 n_{z}+1$. This is used to avoid risks associated with using the unscented transform in high dimensions, such as the risk of estimating non-positive definite covariance matrices and a mean situated far away from each propagated sigma point.

By propagating each sigma point through the mapping (15), we receive the sigma point sets

$$
\left\{\mathbf{R}_{k}^{i}, u_{k}^{i}\right\}_{i=1}^{2 n_{z}+1}, \quad\left\{\boldsymbol{\Sigma}_{k}^{i}, u_{k}^{i}\right\}_{i=1}^{2 n_{z}+1}
$$

where $\mathbf{R}_{k}^{i}$ describe the reflector positions of the $i^{t h}$ sigma point in observation space and $\boldsymbol{\Sigma}_{k}^{i}$ their expected signal power. From (42) we can form estimates of the first two moments of $\mathbf{r}_{k} \mid \mathbf{Y}_{k-1}$ according to

$$
\begin{aligned}
\hat{\mathbf{r}}_{k \mid k-1} & \approx \sum_{i} u_{k}^{i} \mathbf{R}_{k}^{i} \\
\mathbf{P}_{\mathbf{r r}} & \approx \sum_{i} u_{k}^{i}\left(\mathbf{R}_{k}^{i}-\hat{\mathbf{r}}_{k \mid k-1}\right)\left(\mathbf{R}_{k}^{i}-\hat{\mathbf{r}}_{k \mid k-1}\right)^{T}
\end{aligned}
$$

where $\hat{\mathbf{r}}_{k \mid k-1}$ is the estimate of the reflector position. The covariance matrix, $\mathbf{P}_{\mathbf{r r}}$, has the following structure

$$
\mathbf{P}_{\mathbf{r r}}=\left[\begin{array}{cccc}
\mathbf{P}_{\mathbf{r r}}^{11} & \mathbf{P}_{\mathbf{r r}}^{12} & \ldots & \mathbf{P}_{\mathbf{r r}}^{1 n_{r}} \\
\mathbf{P}_{\mathbf{r r}}^{21} & \mathbf{P}_{\mathbf{r r}}^{22} & \ldots & \mathbf{P}_{\mathbf{r r}}^{2 n_{r}} \\
\vdots & \vdots & \ddots & \vdots \\
\mathbf{P}_{\mathbf{r r}}^{n_{r} 1} & \ldots & \ldots & \mathbf{P}_{\mathbf{r r}}^{n_{r} n_{r}}
\end{array}\right]
$$

where $n_{r}$ is the total number of reflectors on the vehicles in $\mathbf{z}_{k}$. From $\Sigma_{k}$, the estimated expected signal power is similarly attained as

$$
\hat{\boldsymbol{\sigma}}_{k \mid k-1} \approx \sum_{i} u_{k}^{i} \Sigma_{k}^{i}
$$

which is used in (30) to account for reflector visibility under state uncertainty, primarily in target heading. In practice, we only need to consider those reflectors which are visible, i.e., for which $\hat{\sigma}_{k \mid k-1}^{i}>0$.

From (43), we determine which reflectors belong to the same group. As only reflectors within each group are able to form clusters with each other, we consider the cluster constellations for each group independently. Following the algorithm in Section III-C, we form all possible cluster constellations for each group. Using the mapping (26) we can calculate two of the sought moments, (36) and (37), as

$$
\begin{aligned}
\hat{\mathbf{g}}_{n} & =\frac{1}{N_{n}^{c c}} \sum_{c c=1}^{N_{n}^{c c}} \sum_{l=1}^{L_{n}^{c c}} q_{n, l}^{c c} \hat{\mathbf{c}}_{n, l}^{c c} \\
\mathbf{P}_{\mathbf{g g}}^{n} & =\sum_{c c=1}^{N_{n}^{c c}} \sum_{l=1}^{L_{n}^{c c}} \frac{q_{n, l}^{c c}}{N_{n}^{c c}}\left(\mathbf{P}_{\mathbf{c}_{l} \mathbf{c}_{l}}^{c c, n}+\left(\hat{\mathbf{g}}_{n}-\hat{\mathbf{c}}_{n, l}^{c c}\right)\left(\hat{\mathbf{g}}_{n}-\hat{\mathbf{c}}_{n, l}^{c c}\right)^{T}\right) .
\end{aligned}
$$

Note that the weights, $q_{n, l}^{c c}$, are dependent on the estimated expected amplitudes, $\hat{\boldsymbol{\sigma}}_{k \mid k-1}$, through $P_{n}^{c c}$. Values for $\hat{\mathbf{c}}_{n, l}^{c c}$ and $\mathbf{P}_{\mathbf{c}_{l} \mathbf{c}_{l}}^{c c, n}$ are found using the same approximations as in the derivation of (21) - (22), detailed in Appendix B, and assuming independence between $w_{i}$ and $\mathbf{r}_{k, i}$,

$$
\begin{aligned}
\hat{\mathbf{c}}_{n, l}^{c c} & =\mathrm{E}\left\{\mathbf{c}_{n, l}^{c c} \mid c c, \mathbf{Y}_{k-1}\right\} \approx \sum_{i=1}^{J_{n, l}^{c c}} \bar{w}_{l_{i}} \hat{\mathbf{r}}_{k \mid k-1}^{l_{i}} \\
\mathbf{P}_{\mathbf{c}_{l} \mathbf{c}_{l}}^{c c, n} & =\operatorname{Cov}\left\{\mathbf{c}_{n, l}^{c c} \mid c c, \mathbf{Y}_{k-1}\right\} \approx \\
\sum_{n, j=1}^{J_{n, l}^{c c}} & \left(\mathbf{P}_{\mathbf{r r}}^{l_{i} l_{j}}+\left(\hat{\mathbf{r}}_{k \mid k-1}^{l_{i}}-\hat{\mathbf{c}}_{n, l}^{c c}\right)\left(\hat{\mathbf{r}}_{k \mid k-1}^{l_{j}}-\hat{\mathbf{c}}_{n, l}^{c c}\right)^{T}\right) \\
& \times \mathrm{E}\left\{w_{l_{i}} w_{l_{j}}\right\},
\end{aligned}
$$

where, $l_{1}, \ldots, l_{J_{n, l}^{c c}}$ are the indexes to the reflectors in the cluster under consideration. Using (47), (48) we can form the Gaussian approximation of the $i^{t h}$ predicted measurement from group $n$ as

$$
p\left(\mathbf{y}_{k, n, i}^{t} \mid \mathbf{Y}_{k-1}\right) \approx \mathcal{N}\left(\mathbf{y}_{k, n, i}^{t} ; \hat{\mathbf{g}}_{n}, \mathbf{P}_{\mathbf{g g}}^{n}+\mathbf{W}_{k}\right) .
$$

The additional sought covariance, $\mathbf{P}_{\mathbf{g g}}^{n m}$, assesses the covariance between two detections from the same group or alternatively two detections from different groups. For the filter to be able to perform the state update for all groups jointly, it is important to accurately assess these correlations. Conditioned on $\mathbf{z}_{k}$, it is assumed that these detections are uncorrelated. Hence, the correlation only comes from uncertainty in $\mathbf{z}_{k}$ and we can approximate the group cross covariance as,

$$
\mathbf{P}_{\mathbf{g g}}^{n m}=\sum_{i}^{J_{n}^{c c}} \sum_{j}^{J_{m}^{c c}} \bar{w}_{n_{i}} \bar{w}_{n_{j}} \mathbf{P}_{\mathrm{rr}}^{n_{i} m_{j}},
$$

where $\left\{n_{i}\right\}_{i=1}^{J_{n}^{c c}}$ and $\left\{m_{i}\right\}_{i=1}^{J_{m}^{c c}}$ are the indices of all the reflectors in each group, respectively. The weights, $\bar{w}_{n_{i}}$ and $\bar{w}_{n_{i}}$, are calculated using the assumption that all reflectors in each group are clustered as, for example,

$$
\bar{w}_{n_{i}}=\mathrm{E}\left\{\frac{A_{n_{i}}}{\sum_{j}^{J_{n}^{c c}} A_{n_{j}}}\right\} .
$$

The cross covariance between detections from the same group is simply found when $n=m$.

\section{Data association}

In difference to standard JPDA, the generalized version of the JPDA algorithm proposed here considers the possibility that a single track can generate multiple measurements. To avoid unlikely data association hypotheses, we employ an ellipsoidal gate [3] centered at the group mean using (51). From the gated measurements and knowledge regarding the maximum number of detections generated by group $n\left(\max M_{k, n}^{t}\right)$, it is possible to construct the set of all local hypotheses, i.e., the set of all feasible associations between $\mathbf{y}_{k}$ and group $n$. By combining local hypotheses from all groups in an admissible fashion (such that each detection in $\mathbf{y}_{k}$ is associated to precisely one group, or classified as clutter) we obtain a global hypothesis, described by the vector $\boldsymbol{\lambda}$. The hypothesis probability can be expressed as

$$
\operatorname{Pr}\left\{\boldsymbol{\lambda} \mid \mathbf{Y}_{k}\right\} \propto p\left(\mathbf{y}_{k} \mid \boldsymbol{\lambda}, \mathbf{Y}_{k-1}\right) \operatorname{Pr}\left\{\boldsymbol{\lambda} \mid \mathbf{Y}_{k-1}\right\} .
$$


The likelihood of the data association hypothesis is found through the Gaussian approximation (51) and the clutter model (7) as

$$
p\left(\mathbf{y}_{k} \mid \boldsymbol{\lambda}, \mathbf{Y}_{k-1}\right)=\mathcal{N}\left(\mathbf{y}_{k}^{\boldsymbol{\lambda}}, \hat{\mathbf{y}}_{k \mid k-1}^{\boldsymbol{\lambda}}, \mathbf{P}_{\mathbf{y y}}^{\boldsymbol{\lambda}}\right)\left(\frac{1}{V}\right)^{M_{k}^{c}} .
$$

where

$$
\begin{aligned}
\hat{\mathbf{y}}_{k \mid k-1}^{\boldsymbol{\lambda}} & =\left[\left(\hat{\mathbf{g}}_{\boldsymbol{\lambda}\left(j_{1}\right)}\right)^{T},\left(\hat{\mathbf{g}}_{\boldsymbol{\lambda}\left(j_{2}\right)}\right)^{T}, \ldots,\left(\hat{\mathbf{g}}_{\boldsymbol{\lambda}\left(j_{m}\right)}\right)^{T}\right]^{T} \\
\mathbf{y}_{k}^{\boldsymbol{\lambda}} & =\left[\left(\mathbf{y}_{k}^{\boldsymbol{\lambda}\left(j_{1}\right)}\right)^{T},\left(\mathbf{y}_{k}^{\boldsymbol{\lambda}\left(j_{2}\right)}\right)^{T}, \ldots,\left(\mathbf{y}_{k}^{\boldsymbol{\lambda}\left(j_{m}\right)}\right)^{T}\right]^{T}
\end{aligned}
$$

for $\left\{j_{1}, \ldots, j_{m}\right\}=\{j: \boldsymbol{\lambda}(j) \neq 0\}$, and similarly $\mathbf{P}_{\mathbf{y} \mathbf{y}}^{\boldsymbol{\lambda}}$ is constructed as

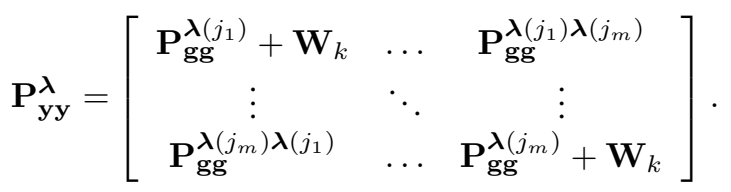

The expected signal component of the group $n, \hat{\mathrm{g}}_{n}$, used in (56) is found in (47). Expressions for the covariance components in (58) are found in (48) for the group covariance, $\mathbf{P}_{\mathrm{gg}}^{n}$, and in (52) for the cross covariances between group $n$ and $m, \mathbf{P}_{\mathbf{g g}}^{n m}$.

The data association vector, $\boldsymbol{\lambda}$, provides perfect knowledge regarding the number of clutter detections, $M_{k}^{c}$, and the number of detections from group $n, M_{k, n}^{t}$. Hence, the prior probability for the association vector in (54) can be partitioned as

$$
\begin{aligned}
\operatorname{Pr}\left\{\boldsymbol{\lambda} \mid \mathbf{Y}_{k-1}\right\} & =\operatorname{Pr}\left\{\boldsymbol{\lambda}, \mathbf{M}_{k}^{t}, M_{k}^{c} \mid \mathbf{Y}_{k-1}\right\} \\
& =\operatorname{Pr}\left\{\boldsymbol{\lambda} \mid \mathbf{M}_{k}^{t}, M_{k}^{c}\right\} \operatorname{Pr}\left\{M_{k}^{c}\right\} \operatorname{Pr}\left\{\mathbf{M}_{k}^{t} \mid \mathbf{Y}_{k-1}\right\},
\end{aligned}
$$

where $\mathbf{M}_{k}^{t}=\left[\begin{array}{lll}M_{k, 1}^{t} & \ldots & M_{k, N_{k}^{g}}^{t}\end{array}\right]^{T}$ and $N_{k}^{g}$ is number of groups. As $M_{k}^{c}$ is assumed to be Poisson distributed, we have

$$
\operatorname{Pr}\left\{M_{k}^{c}\right\}=(\mu V)^{M_{k}^{c}} \exp (-\mu V) / M_{k}^{c} !
$$

Furthermore, $\operatorname{Pr}\left\{\boldsymbol{\lambda} \mid \mathbf{M}_{k}^{t}, M_{k}^{c}\right\}$ is found using combinatorics,

$$
\operatorname{Pr}\left\{\boldsymbol{\lambda} \mid \mathbf{M}_{k}^{t}, M_{k}^{c}\right\}=\prod_{n=1}^{M_{k}^{g}}\left(\begin{array}{c}
M_{k}-\sum_{m=1}^{n-1} M_{k, m}^{t} \\
M_{k, n}^{t}
\end{array}\right)^{-1} .
$$

Finally, the probability of the total number of target detections,

$$
\operatorname{Pr}\left\{\mathbf{M}_{k}^{t} \mid \mathbf{Y}_{k-1}\right\}=\prod_{n=1}^{N_{k}^{g}} \operatorname{Pr}\left\{M_{k, n}^{t} \mid \mathbf{Y}_{k-1}\right\}
$$

where $\operatorname{Pr}\left\{M_{k, n}^{t} \mid \mathbf{Y}_{k-1}\right\}$ is approximated using estimated expected signal amplitude, $\hat{\boldsymbol{\sigma}}_{k \mid k-1}$ in (30).

\section{Measurement update}

To perform the measurement update defined by (34) - (35), we first need to construct the included entities. The predicted mean and covariance are already given in Section IV-A and $\hat{\mathbf{y}}_{k \mid k-1}^{\lambda}$ and $\mathbf{P}_{\mathbf{y y}}^{\lambda}$ are given by (56) and (58), respectively. However, the cross covariance $\mathbf{P}_{\mathbf{z y}}^{\lambda}$ needs to be estimated.
Using the sigma point sets in (41) and (42), $\mathbf{P}_{\mathbf{z r}}=$ $\operatorname{Cov}\left\{\mathbf{z}_{k}, \mathbf{r}_{k} \mid \mathbf{Y}_{k-1}\right\}$ can be estimated as

$$
\mathbf{P}_{\mathbf{z r}} \approx \sum_{i} u_{k}^{i}\left(\mathbf{Z}_{k}^{i}-\hat{\mathbf{z}}_{k \mid k-1}\right)\left(\mathbf{R}_{k}^{i}-\hat{\mathbf{r}}_{k \mid k-1}\right)^{T} .
$$

From (63), $\mathbf{P}_{\mathbf{z y}}^{\lambda}$ is calculated in three steps. First, approximating the relation between reflectors and clusters as in (21) we find

$$
\mathbf{P}_{\mathbf{z} \mathbf{c}_{l}}^{c c, n}=\sum_{i}^{N} \bar{w}_{l_{i}} \mathbf{P}_{\mathbf{z r}}^{l_{i}}
$$

where $l_{i}$ lists all reflectors in cluster $l$ in cluster constellation $c c$. Using (64) and (28), it is easy to find

$$
\mathbf{P}_{\mathbf{z g}}^{n}=\frac{1}{N_{n}^{c c}} \sum_{c c=1}^{N_{n}^{c c}} \sum_{l=1}^{L_{n}^{c c}} q_{n, l}^{c c} \mathbf{P}_{\mathbf{z} \mathbf{c}_{l}}^{c c, n},
$$

from which we can finally construct the sought cross covariance, $\mathbf{P}_{\mathbf{z y}}^{\lambda}$, as

$$
\mathbf{P}_{\mathbf{z y}}^{\boldsymbol{\lambda}}=\left[\mathbf{P}_{\mathbf{z g}}^{\boldsymbol{\lambda}\left(j_{1}\right)}, \ldots, \mathbf{P}_{\mathbf{z g}}^{\boldsymbol{\lambda}\left(j_{m}\right)}\right],
$$

for $\left\{j_{1}, \ldots, j_{m}\right\}=\{j: \boldsymbol{\lambda}(j) \neq 0\}$.

The posterior mean and covariance estimates under data association hypothesis, $\lambda$, are found through inserting (56), (66) and (58) into (34)-(35). The posterior density (33) is a weighted sum of the posterior densities for all $\boldsymbol{\lambda}$ weighted by (54). The mean and covariance of a Gaussian mixture model are readily calculated though moment matching, see e.g. [3]. This concludes the derivation of the filter framework for estimating the position of vehicles using possible unresolved radar detections.

\section{Evaluation}

In this section we compare the proposed extended target model, denoted $\mathcal{M}_{1}$, with that of a point source model (basic model), $\mathcal{M}_{2}$, similar to those presently used in the automotive industry. The evaluation is performed in two steps, First, we compare the ability the models to explain radar observations. Second, we compare the estimation error, $\mathbf{e}_{k}^{i}=$ $\left\|\mathrm{E}\left\{\mathbf{z}_{k}^{i} \mid \mathbf{Y}_{k}, \mathcal{M}_{n}\right\}-\mathbf{z}_{k}^{i}\right\|_{2}$, of the tracking system derived in Section IV with one based on $\mathcal{M}_{2}$. The evaluation is limited to single target vehicle scenarios, i.e. $\mathbf{z}_{k}=\left[\left(\mathbf{z}_{k}^{h}\right)^{T},\left(\mathbf{z}_{k}^{1}\right)^{T}\right]^{T}$.

For both evaluations, radar observations are collected from three sensors, one long-range radar at $77 \mathrm{GHz}$ (denoted $s_{1}$ ) and two medium-range radars at $24 \mathrm{GHz}$ (denoted $s_{2}$ and $s_{3}$ ), mounted on the host vehicle as illustrated in Fig. 6. Sensor $s_{1}$ has an update rate of $10 \mathrm{~Hz}$, a field of view of $16^{\circ}$ and a detection range of approx. $150 \mathrm{~m}$, whereas $s_{2}$ and $s_{3}$ cover a $150^{\circ}$ field of view up to approx. $70 \mathrm{~m}$ using 13 independent receive beams, each delivering detections every $40 \mathrm{~ms}$. The resolution cell for the two types of sensors are, $\boldsymbol{\Delta}_{d}^{s_{1}}=\left[2 \mathrm{~m}, .5 \mathrm{~m} / \mathrm{s}, 3.5^{\circ}\right]$ and $\boldsymbol{\Delta}_{d}^{s_{2,3}}=[2 \mathrm{~m}, 6 \mathrm{~m} / \mathrm{s}, \infty]$, respectively, where $\boldsymbol{\Delta}_{d}^{s_{2,3}}$ is used to describe the resolution in each of the receive beams of $s_{2}$ and $s_{3}$. The corresponding measurement noise covariance for a point target is specified as $\mathbf{W}_{k}^{s_{1}}=\operatorname{diag}\left(\left[.4,2, \frac{1 \pi}{180}\right]\right)^{2}$ and $\mathbf{W}_{k}^{s_{2,3}}=\operatorname{diag}\left(\left[.4, .5, \frac{1.2 \pi}{180}\right]\right)^{2}$. Target vehicle reference 


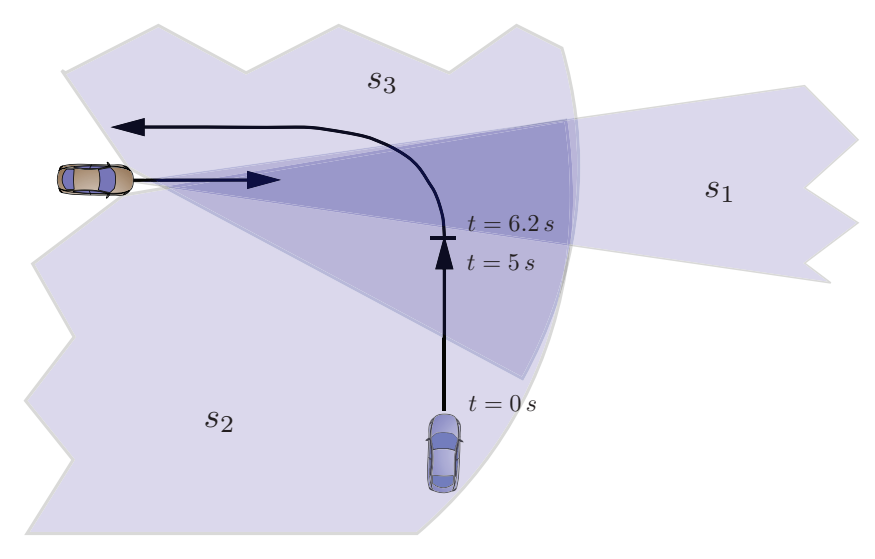

Figure 6. Host vehicle equipped with three radar sensors, one mechanically scanned $77 \mathrm{Ghz}$ long range radar, denoted $s_{1}$, and two medium range $24 \mathrm{GHz}$ radars looking to the right and left, denoted $s_{2}$ and $s_{3}$, respectively. In the evaluated tracking scenario the host vehicle is traveling at constant speed at a straight path. The target vehicle drives at a crossing path stopping in front of the host vehicle before making a left turn.

position, $\mathbf{z}_{k}^{i}$, and host vehicle position measurements are acquired using accurate DGPS measurements. We proceed by introducing the point source model, then explain the two comparisons and their results respectively.

\section{A. Point source model}

To evaluate the tracking performance gained in terms of estimation error by considering the vehicles as extended objects, we compare our tracking system with one based on a point source model. To make the comparison as fair as possible, we use the same state parametrization and both models exploit knowledge regarding the physical dimension of the observed vehicle. Given $\mathbf{z}_{k}^{1}$ the model compensates for offset errors by positioning the expected target measurement, $\hat{\mathbf{y}}_{\mathcal{M}_{2}}^{1}$, on the intersection between the line of sight between the radar sensor and $\left(\zeta_{x, k}^{1}, \zeta_{x, k}^{1}\right)$ and the vehicle frame. Using this model we design a probabilistic data association filter (PDAF) [28], where only one measurement may originate from the target and the presence of multiple measurements are modelled as clutter described by a homogeneous Poisson process.

\section{B. Sensor model comparison}

The ability to explain a set of given observations can be compared by evaluating the log-likelihood ratio

$$
\ell\left(\mathbf{y}_{k}, \mathbf{z}_{k}\right)=\log \left(\frac{p\left(\mathbf{y}_{k} \mid \mathbf{z}_{k}, \mathcal{M}_{1}\right)}{p\left(\mathbf{y}_{k} \mid \mathbf{z}_{k}, \mathcal{M}_{2}\right)}\right)
$$

where the model specific likelihood functions can be partitioned as

$p\left(\mathbf{y}_{k} \mid \mathbf{z}_{k}, \mathcal{M}_{i}\right)=\sum_{\boldsymbol{\lambda}_{k} \in \mathcal{L}_{k}} p\left(\mathbf{y}_{k} \mid \boldsymbol{\lambda}_{k}, \mathbf{z}_{k}, \mathcal{M}_{i}\right) P\left\{\boldsymbol{\lambda}_{k} \mid \mathbf{z}_{k}, \mathcal{M}_{i}\right\}$.

In the following sections, we present expressions for $p\left(\mathbf{y}_{k} \mid \boldsymbol{\lambda}_{k}, \mathbf{z}_{k}, \mathcal{M}_{i}\right)$ and $P\left\{\boldsymbol{\lambda}_{k} \mid \mathbf{z}_{k}, \mathcal{M}_{i}\right\}$ for the different models as well as the evaluation of (67) for radar measurements from two types of radar sensors.
1) Likelihood function: The likelihood ratio test is commonly used to compare hypotheses, in this case which model is more likely to have produced the radar measurements. The test is reasonable if the number of tuning parameters are the same for the compared models. For our proposed sensor model, $\mathcal{M}_{1},(68)$ is given by (55) and (59), with the minor difference that we do not have to integrate over $\mathbf{z}_{k}$. The corresponding densities for $\mathcal{M}_{2}$ are obtained similarly as

$$
\begin{gathered}
p\left(\mathbf{y}_{k} \mid \boldsymbol{\lambda}_{k}, \mathbf{z}_{k}, \mathcal{M}_{2}\right)= \begin{cases}\mathcal{N}\left(\mathbf{y}_{k}^{j} ; \hat{\mathbf{y}}_{\mathcal{M}_{2}}^{1}, \mathbf{W}_{k}\right)\left(\frac{1}{V}\right)^{M_{k}^{c}} & : M_{k}^{t}=1 \\
\left(\frac{1}{V}\right)^{M_{k}^{c}} & : M_{k}^{t}=0\end{cases} \\
\operatorname{Pr}\left\{\boldsymbol{\lambda}_{k} \mid \mathbf{z}_{k}, \mathcal{M}_{2}\right\}= \begin{cases}\frac{P_{D}}{M_{k}} \frac{e^{-\mu V}(\mu V)^{M_{k}^{c}}}{M_{k}^{c} !} & : M_{k}^{t}=1 \\
\left(1-P_{D}\right) \frac{e^{-\mu V}(\mu V)^{M_{k}^{c}}}{M_{k}^{c !}} & : M_{k}^{t}=0 .\end{cases}
\end{gathered}
$$

The probability of detection is modelled in the same way for both models and the clutter intensity, $\mu$, is estimated from data using nonparametric PDA [29]. Note that the same parameters are used for tuning both models, i.e., the measurement noise covariance and the probability of detection. All other parameters are taken directly from the sensor specification.

2) Results: The log-likelihood ratio (67) is evaluated using radar measurements from two sensors of different types, $s_{1}$ and $s_{2}$. The data is collected while the host vehicle drives straight towards the target vehicle at an angle of $18^{\circ}$ (offset from the side of the vehicle) starting at a distance of $40 \mathrm{~m}$. A scatter plot of the collected data is illustrated in Fig. 7a.

Figures $7 \mathrm{~b}$ and $7 \mathrm{c}$ display (67) evaluated for measurements delivered by sensor $s_{1}$ and $s_{2}$, respectively. Both figures show a clear advantage in favour of our proposed model. This is especially clear for sensor $s_{1}$ which has higher resolution than sensor $s_{2}$ and where we often receive multiple detections neatly concentrated to the front and rear wheel housings, see Fig. 7a. For sensor $s_{2}$, the detections are spread along the side of the target vehicle (some even positioned outside the vehicle frame). This behavior is also modelled in $\mathcal{M}_{1}$ but the advantage is not as dominant as in the case of sensor $s_{1}$.

\section{Tracking filter comparison}

The tracking filters based on $\mathcal{M}_{1}$ and $\mathcal{M}_{2}$ are evaluated using data from $s_{1}, s_{2}$ and $s_{3}$ in the scenario depicted in Fig. 6. This particular scenario is chosen, both because it is a relevant scenario for active safety systems addressing intersection accidents [30] and because it is challenging for a radar based tracking system.

In the evaluation, both filters are initiated in the reference state, $\mathbf{z}_{0}^{1}$, provided by the DGPS system with initial covariance $\mathbf{P}_{0}=\operatorname{diag}\left(\left[1,1, \frac{3 \pi}{180}, .5, .001,1.5\right]\right)^{2}$ and use the same process noise parameters, $\sigma_{\ddot{v}}^{2}=9$ and $\sigma_{\dot{c}}^{2}=\frac{1}{70}$. The filter implementation for our proposed model is given in Section IV and for the point source model we employ a standard UKF.

The result of the comparison is shown in Fig. 8 in terms of absolute longitudinal and lateral positioning error, $e_{x}$ and $e_{y}$, in the reference vehicle coordinate frame as well as absolute velocity error, $e_{v}$, and heading angle error, $e_{\psi}$, in the global coordinate frame. The result indicates a clear advantage for $\mathcal{M}_{1}$ in terms of accurate and stable positioning of the target vehicle, as well as velocity and heading estimates. Worth 


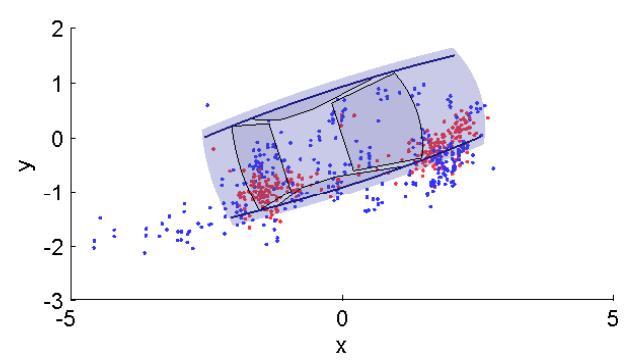

(a) Scatter plot of the evaluated target measurements from the $s_{1}$ (red) and $s_{2}$ (blue) radars.

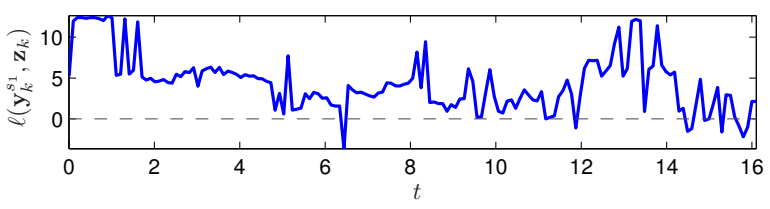

(b) Log-likelihood ratio $s_{1}$

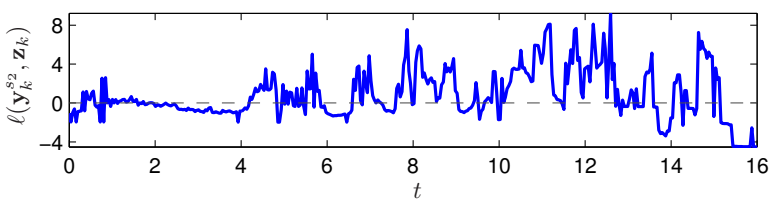

(c) Log-likelihood ratio $s_{2}$

Figure 7. Log-likelihood ratio comparison between our model, $\mathcal{M}_{1}$, and the simpler point source model, $\mathcal{M}_{2}$.

noting is the later part of the scenario, $t \in[7,10] s$, where the two vehicles are close and many of the features on the reference vehicle are resolved. In this part of the scenario the target vehicle starts to turn, something that confuses the simpler point source model while our model still manages to position the vehicle well. Additionally, the jump in the heading estimation error at $t \approx 9.8 \mathrm{~s}$ is explained by the filters only receiving measurements from the rear part of the vehicle for some updates. As measurements again appear from the front of the vehicle, it is clear that our proposed model is able to take more advantage of the new information to deduce the heading of the vehicle more accurately than the point source model.

\section{CONCLUSION}

In this paper we have proposed an accurate and tractable radar sensor model capable of describing both multiple detections from a vehicle and their relation to the limited sensor resolution. Furthermore, we have developed a framework for tracking vehicles based on this model. The evaluation of the sensor model shows that our model is clearly better than the reference model at describing the vehicle radar detections from the two evaluated sensors. Additionally, the evaluation of the tracking performance indicates substantial benefits using our model compared to the reference model.

The reference model is notably simpler than the proposed one, and clearly penalized in the evaluation when multiple features are resolved. However, it is presently used in many systems and our comparison show that a widely used family
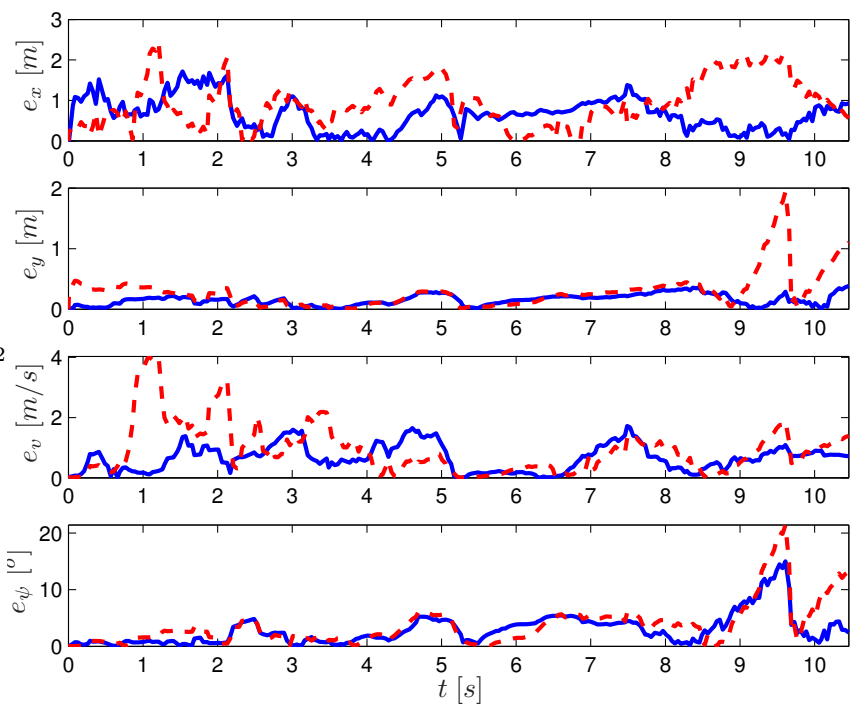

Figure 8. Comparison of the absolute estimation error from our tracking framework (solid blue) and the point source model (dashed red) for longitudinal and lateral positioning error in the reference vehicle coordinate frame, $e_{x}$ and $e_{y}$, as well as absolute velocity error, $e_{v}$, and heading angle error, $e_{\psi}$, in the global coordinate frame.

of tracking frameworks can be adapted to incorporate the new measurement model, with improved performance as a result.

By formally including sensor resolution in the model, it can be used for a wide range of sensors and targets by changing appropriate parameters according to the sensor specification. This feature is of most importance to the automotive industry as it allows for sensors to be more easily replaced or updated.

\section{APPENDIX A \\ REFLECTOR MAPPING}

The reflector mapping is divided into two parts; first the reflector is positioned in the observation space, second, the the expected signal amplitude is modelled.

\section{A. Reflector position}

Assuming that point reflector with index $i$ is positioned at $\mathbf{x}_{i}=\left(x_{i}, y_{i}\right)$ in a local coordinate system with the origin in the center of target vehicle $j$. The global position of the reflector is then given by

$$
\left[\begin{array}{l}
\zeta_{x}^{j, i} \\
\zeta_{y}^{j, i}
\end{array}\right]=\left[\begin{array}{l}
\zeta_{x_{k}}^{j} \\
\zeta_{y_{k}}^{j}
\end{array}\right]+\mathbf{R}\left(\psi_{k}^{j}\right)\left[\begin{array}{l}
x_{i} \\
y_{i}
\end{array}\right]
$$

where $\mathbf{R}(\cdot)$ is a $2 \times 2$ rotational matrix. Similarly, assuming that sensor, $s$, is mounted on the host vehicle at $\mathbf{x}_{s}=\left(x_{s}, y_{s}\right)$ and with an angle of $\psi_{s}$, the global position of the sensor is defined as

$$
\left[\begin{array}{l}
\zeta_{x}^{s} \\
\zeta_{y}^{s}
\end{array}\right]=\left[\begin{array}{l}
\zeta_{x_{k}}^{h} \\
\zeta_{y_{k}}^{h}
\end{array}\right]+\mathbf{R}\left(\psi_{k}^{h}\right)\left[\begin{array}{l}
x_{s} \\
y_{s}
\end{array}\right]
$$

Additionally, we define the relative angle between reflector $i$ and sensor $s$ according to

$$
\alpha_{i, s}=\arctan \left(\frac{\zeta_{y}^{j, i}-\zeta_{y}^{s}}{\zeta_{x}^{j, i}-\zeta_{x}^{s}}\right)
$$


Using these relations, the mapping $\mathbf{z}_{k} \stackrel{\mathcal{R}_{i}(\cdot)}{\longrightarrow} \mathbf{r}_{i}$ is defined as

$$
\begin{aligned}
& r_{i}=\sqrt{\left(\zeta_{x}^{j, i}-\zeta_{x}^{s}\right)^{2}+\left(\zeta_{y}^{j, i}-\zeta_{y}^{s}\right)^{2}} \\
& \dot{r}_{i}=\left(v_{k}^{j} \cos \left(\psi_{k}^{l}-\alpha_{i, s}\right)+v_{\perp}^{j} \cos \left(\frac{\pi}{2}+\arg \left(\mathbf{x}_{i}\right)-\alpha_{i, s}\right)\right) \\
& -\left(v_{k}^{h} \cos \left(\psi_{k}^{h}-\alpha_{i, s}\right)+v_{\perp}^{h} \cos \left(\frac{\pi}{2}+\arg \left(\mathbf{x}_{s}\right)-\alpha_{i, s}\right)\right) \\
& \phi_{i}=\alpha_{i, s}-\psi_{k}^{h}-\psi_{s}
\end{aligned}
$$

where $v_{\perp}^{j}=v_{k}^{j} c_{k}^{j}\left\|\mathbf{x}_{i}\right\|$ and $v_{\perp}^{h}=v_{k}^{h} c_{k}^{h}\left\|\mathbf{x}_{s}\right\|$ are the velocity component due to rotation of the target and host vehicle, respectively.

\section{B. Signal amplitude}

The signal power of the sensor is characterized by two functions, the reciprocal antenna gain pattern, $A_{a}(\phi)$, and the signal attenuation, $A_{r}(r)$. Associated with each reflector is a visibility function, $\nu_{\sigma}^{i}\left(\alpha_{i, s}, \psi_{k}^{j}\right)$ dependent on the relative angle between the observing sensor and the reflector and the heading of the target vehicle. Using these models, the expected return amplitude of reflector $i$ is calculated as,

$$
\sigma_{i}=A_{a}\left(\phi_{i}\right) A_{r}\left(r_{i}\right) \nu_{\sigma}^{i}\left(\alpha_{i, s}, \psi_{k}^{j}\right) .
$$

\section{APPENDIX B}

\section{GAUSSIAN CLUSTER DENSITY APPROXIMATION}

In this section we derive the gaussian approximation of the cluster density in (21) and (22). Let a cluster $i$ consist of $N$ reflectors positioned at $\mathbf{r}_{1}, \mathbf{r}_{2}, \ldots, \mathbf{r}_{N}$ in measurement space. According to (18) the signal component of the cluster is given by

$$
\mathbf{c}_{i}=\sum_{n=1}^{N} w_{n} \mathbf{r}_{n},
$$

where in this case the reflector positions, $\mathbf{r}_{n}$, are known whereas the weights, $w_{n}$, are stochastic. The weights are expressed in terms of the received signal amplitudes of each reflector, $A_{n}$,

$$
w_{n}=\frac{A_{n}}{\sum_{m=1}^{N} A_{m}} .
$$

where

$$
A_{n} \sim \operatorname{Rayleigh}\left(\sigma_{n}\right) .
$$

To find a Gaussian approximation of cluster density we need to find the first two moments of $\mathbf{c}_{i}$.

\section{A. Mean approximation}

The mean of $\mathbf{c}_{i}$ is

$$
\overline{\mathbf{c}}_{i}=\mathrm{E}\left\{\mathbf{c}_{i}\right\}=\sum_{n=1}^{N} \bar{w}_{n} \mathbf{r}_{n} .
$$

where $\bar{w}_{n}=\mathrm{E}\left\{w_{n}\right\}$ is not trivial to express. However, monteCarlo simulations indicate that the approximation

$$
\bar{w}_{n} \approx \frac{\bar{A}_{n}}{\sum_{m=1}^{N} \bar{A}_{m}}
$$

where

$$
\bar{A}_{n}=\mathrm{E}\left\{A_{n}\right\}=\sigma_{n} \sqrt{\frac{\pi}{2}},
$$

yields a reasonable approximation of (76).

\section{B. Covariance approximation}

The remaining difficulty is to approximate the covariance matrix of $\mathbf{c}_{i}$,

$$
\mathbf{C}_{i}=E\left\{\left(\mathbf{c}_{i}-\overline{\mathbf{c}}_{i}\right)\left(\mathbf{c}_{i}-\overline{\mathbf{c}}_{i}\right)^{T}\right\}
$$

The first aim is to find a representation which more robust to approximations. For notation, set $\Delta \mathbf{r}_{n}=\mathbf{r}_{n}-\overline{\mathbf{c}_{i}}$ and $\Delta w_{n}=$ $w_{n}-\bar{w}_{n}$. In the following, we will use the relations

$$
\sum_{n=1}^{N} w_{n}=1,
$$

which follows from the definition of $w_{n}$, and

$$
\sum_{n=1}^{N} \bar{w}_{n} \Delta \mathbf{r}_{n}=0
$$

which is clear due to $E\{\mathbf{r}-\overline{\mathbf{r}}\}=0$. These relations and notations yield

$$
\begin{aligned}
\mathbf{C}_{i} & =E\left\{\left(\sum_{n=1}^{N} w_{n}\left(\mathbf{r}_{n}-\overline{\mathbf{r}}\right)\right)\left(\sum_{n=1}^{N} w_{n}\left(\mathbf{r}_{n}-\overline{\mathbf{r}}\right)\right)^{T}\right\} \\
& =E\left\{\left(\sum_{n=1}^{N} w_{n} \Delta \mathbf{r}_{n}\right)\left(\sum_{n=1}^{N} w_{n} \Delta \mathbf{r}_{n}\right)^{T}\right\} \\
& =E\left\{\left(\sum_{n=1}^{N} \Delta w_{n} \Delta \mathbf{r}_{n}\right)\left(\sum_{n=1}^{N} \Delta w_{n} \Delta \mathbf{r}_{n}\right)^{T}\right\} \\
& =\sum_{n=1}^{N} \sum_{m=1}^{N} \Delta \mathbf{r}_{n} \Delta \mathbf{r}_{m}^{T} E\left\{\Delta w_{n} \Delta w_{m}\right\} .
\end{aligned}
$$

Recall that the matrices $\Delta \mathbf{r}_{n} \Delta \mathbf{r}_{m}^{T}$ are known for all indices $n$ and $m$. Hence, we will now strive to find approximations for the scalar factors

$$
\operatorname{Cov}\left\{w_{n}, w_{m}\right\}=E\left\{\Delta w_{n} \Delta w_{m}\right\} .
$$

To this end, we use the Taylor approximation

$$
\begin{aligned}
w_{n} & =\frac{A_{n}}{S_{i}} \\
& \approx \frac{\bar{A}_{n}}{\bar{S}_{i}}+\frac{A_{n}-\bar{A}_{n}}{\bar{S}_{i}}-\frac{\left(S_{i}-\bar{S}_{i}\right) \bar{A}_{n}}{\bar{S}_{i}^{2}} \\
& =\frac{\bar{A}_{n}}{\bar{S}_{i}}+\frac{A_{n}}{\bar{S}_{i}}-\frac{S_{i} \bar{A}_{n}}{\bar{S}_{i}^{2}}
\end{aligned}
$$

where $S_{i}=\sum_{m=1}^{N} A_{m}$ and

$$
\begin{aligned}
\bar{A}_{n} & =E\left\{A_{n}\right\} \\
\bar{S}_{i} & =E\left\{\sum_{m=1}^{N} A_{m}\right\} .
\end{aligned}
$$


Thus, we get

$$
\begin{aligned}
\operatorname{Cov}\left\{w_{n}, w_{m}\right\} & \approx E\left\{\left(\frac{A_{n}}{\bar{S}_{i}}-\frac{S_{i} \bar{A}_{n}}{\bar{S}_{i}^{2}}\right)\left(\frac{A_{m}}{\bar{S}_{i}}-\frac{S_{i} \bar{A}_{m}}{\bar{S}_{i}^{2}}\right)\right\} \\
& =\frac{E\left\{A_{n} A_{m}\right\}}{\bar{S}_{i}^{2}}-\frac{E\left\{A_{n} S_{i}\right\} \bar{A}_{m}}{\bar{S}_{i}^{3}} \\
& -\frac{E\left\{A_{m} S_{i}\right\} \bar{A}_{n}}{\bar{S}_{i}^{3}}+\frac{E\left\{S_{i}^{2}\right\} \bar{A}_{n} \bar{A}_{m}}{\bar{S}_{i}^{4}}
\end{aligned}
$$

To evaluate (87), we essentially only need the relations

$$
\begin{aligned}
E\left\{A_{n}\right\} & =\sigma_{n} \sqrt{\pi / 2} \\
\overline{A_{n}^{2}} & =E\left\{A_{n}^{2}\right\}=2 \sigma_{n}^{2} \\
E\left\{A_{n} A_{m}\right\} & = \begin{cases}E\left\{A_{n}^{2}\right\} & \text { if } n=m \\
E\left\{A_{n}\right\} E\left\{A_{m}\right\} & \text { otherwise. }\end{cases}
\end{aligned}
$$

\section{REFERENCES}

[1] J. Gunnarsson, L. Svensson, L. Danielsson, and F. Bengtsson, “Tracking vehicles using radar detections," in Intelligent Vehicles Symposium, 2007. Proceedings IEEE, Istanbul, June 2007.

[2] W. Koch, "Advanced sensor models: Benefits for target tracking and sensor data fusion," in Multisensor Fusion and Integration for Intelligent Systems, 2006 IEEE International Conference on, sep 2006, pp. 565570.

[3] S. Blackman and R. Popoli, Design and analysis of modern tracking systems. Norwood, MA: Artech House, 1999.

[4] Y. Bar-Shalom and W. D. Blair, Multitarget-Multisensor Tracking Volume III: Applications and Advances. Norwood, MA: Artech House, 2000.

[5] R. Ostrovityanov and F. Basalov, Statistical Theory of Extended Radar Targets. Artech House, 1985

[6] M. Waxman and O. Drummond, "A bibliography of cluster (group) tracking," in Signal and Data Processing of Small Targets 2004. Edited by Drummond, Oliver E. Proceedings of the SPIE, vol. 5428, 2004, pp. $551-560$.

[7] W. Koch and R. Saul, "A Bayesian approach to extended object tracking and tracking of loosely structured target groups," in Information Fusion, 2005 8th International Conference on, vol. 1, 2005.

[8] W. Koch, "On bayesian tracking of extended objects," in Multisensor Fusion and Integration for Intelligent Systems, 2006 IEEE International Conference on, Sept. 2006, pp. 209-216.

[9] K. Gilholm, S. J. Godsill, S. Maskell, and D. J. Salmond, "Poisson models for extended target and group tracking," SPIE, October 2005.

[10] D. F. Crouse, S. Member, M. Guerriero, and P. Willett, "A Critical Look at the PMHT," Journal of Advances in Infor- mation Fusion, 2009.

[11] M. Wieneke and W. Koch, "Probabilistic tracking of multiple extended targets using random matrices," in Society of Photo-Optical Instrumentation Engineers (SPIE) Conference Series, ser. Presented at the Society of Photo-Optical Instrumentation Engineers (SPIE) Conference, vol. 7698, Apr. 2010

[12] M. Bühren and B. Yang, "Simulation of automotive radar target lists using a novel approach of object representation," in IEEE Intelligent Vehicles Symposium (IV), Tokyo, Japan, June 2006.

[13] W. Koch and G. Van Keuk, "Multiple hypothesis track maintenance with possibly unresolved measurements," Aerospace and Electronic Systems, IEEE Transactions on, vol. 33, no. 3, pp. 883-892, July 1997.

[14] H. Blom and E. Bloem, "Bayesian tracking of two possibly unresolved maneuvering targets," Aerospace and Electronic Systems, IEEE Transactions on, vol. 43, no. 2, pp. 612-627, April 2007.

[15] — "Hybrid SIR joint particle filtering under limited sensor resolution," in Information Fusion, 2007 10th International Conference on, 2007, pp. $1-8$

[16] S. Jeong and J. Tugnait, "Tracking of two targets in clutter with possibly unresolved measurements," Aerospace and Electronic Systems, IEEE Transactions on, vol. 44, no. 2, pp. 748-765, April 2008.

[17] K.-C. Chang and Y. Bar-Shalom, "Joint probabilistic data association for multitarget tracking with possibly unresolved measurements and maneuvers," Automatic Control, IEEE Transactions on, vol. 29, no. 7, pp. 585-594, Jul 1984.
[18] L. Ng and Y. Bar-Shalom, "Model of unresolvable measurements for multitarget tracking," in Preceedings of the OCEANS 1981 conference, Sept 1981, pp. 982-987.

[19] S. Julier and J. Uhlmann, "Unscented filtering and nonlinear estimation," Proceedings of the IEEE, vol. 92, no. 3, 2004

[20] T. Fortmann, Y. Bar-Shalom, and M. Scheffe, "Sonar tracking of multiple targets using joint probabilistic data association," IEEE Journal of Oceanic Engineering, vol. 8, July 1983.

[21] F. E. Daum and R. J. Fitzgerald, "Importance of resolution in multiple-target tracking," Signal and Data Processing of Small Targets 1994, vol. 2235, no. 1, pp. 329-338, 1994. [Online]. Available: http://link.aip.org/link/?PSI/2235/329/1

[22] M. Arulampalam, N. Gordon, M. Orton, and B. Ristic, "A variable structure multiple model particle filter for gmti tracking," Proceedings of the Fifth International Conference on Information Fusion. FUSION 2002. (IEEE Cat.No.02EX5997), vol. vol.2, pp. 927 - 34, 2002.

[23] P. Swerling, "Probability of detection for fluctuating targets," Information Theory, IRE Transactions on, vol. 6, no. 2, pp. 269-308, 1960.

[24] J. Dunn and D. Howard, "Radar target amplitude, angle, and Doppler scintillation from analysis of the echo signal propagating in space," IEEE Transactions on Microwave Theory and Techniques, vol. 16, no. 9, pp. 715-728, 1968

[25] B. Ristic, S. Arulampalam, and N. Gordon, Beyond the Kalman filter: particle filters for tracking applications. Norwood, MA: Artech House, 2004.

[26] D. Reid, "An algorithm for tracking multiple targets," Automatic Control, IEEE Transactions on, vol. 24, no. 6, pp. 843-854, Dec 1979.

[27] R. Kalman, "A new approach to linear filtering and prediction problems," Trans. ASME, Ser. D. Journal Basic Eng., vol. 82, pp. 24-45, 1960.

[28] Y. Bar-Shalom and E. Tse, "Tracking in a cluttered environment with probabilistic data association," Automatica, vol. 11, September 1975.

[29] Y. Bar-Shalom and T. Fortmann, Tracking and data association. Academic-Press, Boston, 1988.

[30] M. Brännström, E. Coelingh, and J. Sjöberg, "Model-based threat assessment for avoiding arbitrary vehicle collisions," Intelligent Transportation Systems, IEEE Transactions on, vol. 11, no. 3, pp. 658-669, 2010.

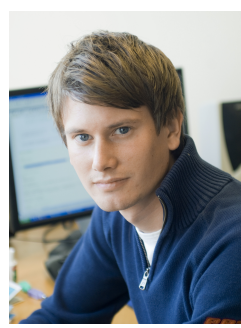

Lars Hammarstrand was born in Landvetter, Sweden in 1979. He received his M.Sc. and Ph.D. degree in electrical engineering from Chalmers University of Technology, Gothenburg, Sweden, in 2004 and 2010, respectively.

From 2004 to 2011, he was with the Active Safety and Chassis Department at Volvo Car Corporation, Gothenburg, conducting research on tracking and sensor data fusion methods for active safety systems. Currently, Lars is a Postdoctoral Research Fellow at the Signal Processing group at Chalmers University of Technology where his main research interests are in the fields of estimation, sensor fusion and radar sensor modeling, especially with application to active safety systems.

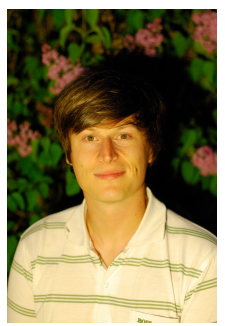

Fredrik Sandblom received the M.S. degree in electrical engineering in 2004 and the Licentiate of Engineering degree in 2008, both from Chalmers University of Technology, Gothenburg, Sweden.

Since 2005 he has been with the Volvo group, working with active safety system research as a part of his Ph.D. studies - he will defend his thesis in December 2011. His research interests concern object tracking and sensor data fusion, particularly sigma-point methods for estimating statistical moments and their application to recursive filtering. 


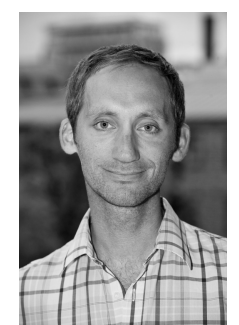

Lennart Svensson was born in Älvängen, Sweden in 1976. He received the M.S. degree in electrical engineering in 1999 and the Ph.D. degree in 2004, both from Chalmers University of Technology, Gothenburg, Sweden.

$\mathrm{He}$ is currently an Associate Professor at the Signal Processing group, again at Chalmers University of Technology. His research interests include Bayesian inference in general, and nonlinear filtering and tracking in particular.

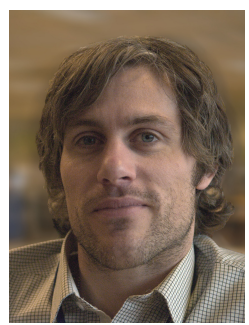

Joakim Sörstedt was born in Skara, Sweden, on April 3, 1976. He received the M.S. degree in electrical engineering in 2001 and the Ph.D. degree in 2007, both from Chalmers University of Technology, Gothenburg, Sweden.

Since 2007 he has been with the Volvo Car Corporation where he has worked on developing active safety systems. His research interests are in the area of signal processing; specifically subspacebased estimation methods and object tracking. 